OPEN ACCESS

Edited by:

Joao Batista Teixeira da Rocha,

Federal University of Santa

Maria, Brazi

Reviewed by:

Gabriele Stocco,

University of Trieste, Italy

Kevin Gerrish,

National Institute of Environmental Health Sciences (NIEHS),

United States

*Correspondence:

Julia do Amaral Gomes juuliadoamara/@gmail.com

Hedvig Marie Egeland Nordeng

h.m.e.nordeng@farmasi.uio.no

Specialty section

This article was submitted to

Toxicogenomics,

a section of the journal

Frontiers in Genetics

Received: 23 December 2020

Accepted: 19 March 2021

Published: 27 April 2021

Citation:

Gomes JA, Olstad EW, Kowalski TW

Gervin K, Vianna FSL,

Schüler-Faccini $L$ and Nordeng HME (2021) Genetic Susceptibility to Drug

Teratogenicity: A Systematic Literature

Review. Front. Genet. 12:645555.

doi: 10.3389/fgene.2021.645555

\title{
Genetic Susceptibility to Drug Teratogenicity: A Systematic Literature Review
}

\author{
Julia do Amaral Gomes ${ }^{1,2,3,4 *}$, Emilie Willoch Olstad ${ }^{5,6}$, Thayne Woycinck Kowalski ${ }^{1,3,4,7}$, \\ Kristina Gervin ${ }^{5,6,8}$, Fernanda Sales Luiz Vianna ${ }^{1,2,3,4}$, Lavínia Schüler-Faccini ${ }^{1,2,4}$ and \\ Hedvig Marie Egeland Nordeng ${ }^{5,6,9 *}$
}

${ }_{1}^{1}$ Programa de Pós-Graduação em Genética e Biologia Molecular (PPGBM), Departamento de Genética, Universidade Federal do Rio Grande do Sul (UFRGS), Porto Alegre, Brazil, ${ }^{2}$ Sistema Nacional de Informação sobre Agentes Teratogênicos (SIAT), Serviço de Genética Médica, Hospital de Clínicas de Porto Alegre (HCPA), Porto Alegre, Brazil, ${ }^{3}$ Laboratório de Medicina Genômica, Centro de Pesquisa Experimental, Hospital de Clínicas de Porto Alegre (HCPA), Porto Alegre, Brazil, ${ }^{4}$ Instituto Nacional de Genética Médica Populacional (INAGEMP), Hospital de Clínicas de Porto Alegre (HCPA), Porto Alegre, Brazil, ${ }^{5}$ Pharmacoepidemiology and Drug Safety Research Group, Department of Pharmacy, Faculty of Mathematics and Natural Sciences, University of Oslo, Oslo, Norway, ${ }^{6}$ PharmaTox Strategic Research Initiative, Faculty of Mathematics and Natural Sciences, University of Oslo, Oslo, Norway, ${ }^{7}$ Complexo de Ensino Superior de Cachoeirinha (CESUCA), Cachoeirinha, Brazil, ${ }^{8}$ Division of Clinical Neuroscience, Department of Research and Innovation, Oslo University Hospital, Oslo, Norway, ${ }^{9}$ Department of Child Health and Development, Norwegian Institute of Public Health, Oslo, Norway

Since the 1960s, drugs have been known to cause teratogenic effects in humans. Such teratogenicity has been postulated to be influenced by genetics. The aim of this review was to provide an overview of the current knowledge on genetic susceptibility to drug teratogenicity in humans and reflect on future directions within the field of genetic teratology. We focused on 12 drugs and drug classes with evidence of teratogenic action, as well as 29 drugs and drug classes with conflicting evidence of fetal safety in humans. An extensive literature search was performed in the PubMed and EMBASE databases using terms related to the drugs of interest, congenital anomalies and fetal development abnormalities, and genetic variation and susceptibility. A total of 29 studies were included in the final data extraction. The eligible studies were published between 1999 and 2020 in 10 different countries, and comprised 28 candidate gene and 1 whole-exome sequencing studies. The sample sizes ranged from 20 to 9,774 individuals. Several drugs were investigated, including antidepressants (nine studies), thalidomide (seven studies), antiepileptic drugs (five studies), glucocorticoids (four studies), acetaminophen (two studies), and sex hormones (estrogens, one study; 17-alpha hydroxyprogesterone caproate, one study). The main neonatal phenotypic outcomes included perinatal complications, cardiovascular congenital anomalies, and neurodevelopmental outcomes. The review demonstrated that studies on genetic teratology are generally small, heterogeneous, and exhibit inconsistent results. The most convincing findings were genetic variants in SLC6A4, MTHFR, and NR3C1, which were associated with drug teratogenicity by antidepressants, antiepileptics, and glucocorticoids, respectively. Notably, this review demonstrated the large knowledge gap regarding genetic susceptibility to drug teratogenicity, emphasizing the need for further 
efforts in the field. Future studies may be improved by increasing the sample size and applying genome-wide approaches to promote the interpretation of results. Such studies could support the clinical implementation of genetic screening to provide safer drug use in pregnant women in need of drugs.

Keywords: teratogens, prenatal exposures, pregnancy, congenital anomalies, thalidomide, antidepressant, anticonvulsants, genetic predisposition to disease

\section{INTRODUCTION}

Congenital anomalies are the leading cause of infant mortality in high-income countries and the second most common cause in many middle-income countries (Sitkin et al., 2015). Such conditions emerge during fetal development and can be inherited or influenced by environmental factors, such as medication exposure (Song and Yosypiv, 2011; Harris et al., 2017; Williams et al., 2019). The prescription of drugs during pregnancy is common, with a prevalence of $40 \%$ to $80 \%$ in Western countries (Olesen et al., 1999; Engeland et al., 2008; Bakker et al., 2010). Drugs used by the mother during pregnancy can be teratogenic, causing abnormal development of the embryo or fetus. Although such exposures may be preventable, many maternal diseases need to be treated. For example, in cases of cancer, epilepsy, and autoimmune diseases, a drug with known teratogenic potential may be the most effective one, leaving patients and prescribers in a dilemma with challenging treatment decisions (Ostensen, 1992; Bromley et al., 2017; Momen et al., 2017).

Teratogens do not produce congenital anomalies in all exposed embryos, as recognized by Wilson when he proposed his principles of teratogenesis (Wilson, 1977). These principles are still pivotal in the study of teratogenicity and interpretation of teratogenic risk. First, the principles state that the susceptibility to a teratogenic agent depends on the genotype of both the embryo and the mother, as well as the timing of the exposure and the developmental stage of the embryo. In addition, the teratogenic agents act in particular ways on cells or tissues to cause pathogenesis, and the final indications of abnormal development are death, malformation, growth restriction, or functional disorders. Finally, access to the embryo by environmental teratogens depends on the agent's nature, and as the dosage of the agent increases, manifestation of deviant development also increases (Wilson, 1977).

Although little was known about gene-environment interactions and developmental processes at the time Wilson formulated his principles, recent advances in genetics and developmental biology have led to significant improvements in our understanding of the mechanisms of teratogenesis (Yalcin et al., 2015; Griffiths et al., 2018; Piña et al., 2018). Teratogenic events are complex, but an examination of genetic influences on drug response (pharmacogenetics) may promote our understanding of the molecular mechanisms involved (Cassina et al., 2012). This knowledge can be utilized both to identify susceptible individuals by whom the drug should be avoided, and in designing drugs with therapeutic effects and no or minimal teratogenic potential. No recent review of the literature is available in this research field. Therefore, the aim of this systematic review was to provide an overview of the current knowledge on genetic susceptibility to drug teratogenicity in humans and to elucidate future directions within the field.

\section{MATERIALS AND METHODS}

\section{Selection of Drugs}

Two lists of drugs were created for this review. First, a list of major teratogens was identified based on the book "Drugs during Pregnancy and Lactation: Treatment Options and Risk Assessment" (Schaefer et al., 2014; Appendix 1). This list contains the 12 drugs or classes of drugs that have been recognized across several studies to compromise normal human development in utero. A second list was created including 29 drugs and classes of drugs that may present a teratogenic or fetotoxic risk to humans according to multiple reviews and original studies (Appendix 2). This list was generated using a wide range of references and discussed among the authors until consensus was reached.

\section{Eligibility Criteria}

The studies included in the review were selected based on the participants, intervention/exposure, comparison group, outcome and study design (PICOS) criteria (Richardson et al., 1995; Eriksen and Frandsen, 2018). Participants were defined as mothers and/or their children exposed to a drug during pregnancy (embryonic/fetal period of development) with known fetal outcome(s). The intervention/exposure was defined as exposure to a drug during pregnancy included in Appendices 1 or 2. The comparison group included mothers and/or their children with different genetic backgrounds. The outcome of interest was the differential occurrence or severity of congenital anomalies or developmental abnormalities based on the association of drug exposure and the genetic background of the individuals. The considered study designs were case-control, cohort, and randomized controlled trials of original articles. Our study was performed in adherence to the Preferred Reporting Items for Systematic Reviews and Meta-Analyses guidelines (PRISMA) (Liberati et al., 2009). Studies in English, Portuguese, and Scandinavian were eligible. No restriction on publication date was set.

Studies were excluded if they had a non-eligible study design (e.g., case reports, studies without a comparison group, studies lacking drug exposure, studies including exposure to many indiscernible drugs, studies assessing drug exposure only after birth, studies with no difference in genetic variation across comparison groups, and in vitro or animal model studies), noneligible outcome (e.g., if congenital anomalies or developmental 
abnormalities were not evaluated), or if the publication type was non-eligible (e.g., review article, editorial, or conference abstract).

\section{Search Strategy}

Literature searches were performed in the PubMed and EMBASE databases. These databases were selected for the search, as they are the most complete and comprehensive in the fields of medicine and genetics (Khare et al., 2014). The search strategy included: (1) terms associated with the relevant drugs, (2) terms associated with congenital anomalies or developmental abnormalities, and (3) terms associated with genetic variation and genetic susceptibility. The search terms are available in Appendix 3.

Studies published until November 10, 2020, were included in the review. To ensure full coverage of the literature, we performed additional screening of the reference lists for relevant reviews and eligible studies to discover any research articles not identified in the database searches.

\section{Study Selection}

The list of studies generated after searching each database was compiled in EndNote to remove any duplicates. The studies were then imported into Rayyan, a platform for data management in systematic review projects (Ouzzani et al., 2016). The screening and selection of relevant studies was performed independently in two screening rounds by two reviewers (JAG and EWO). In the first round, articles were screened by the titles and abstracts. In the second round, full texts of the articles selected from the first screening were assessed based on the predefined inclusion and exclusion criteria outlined above. Disagreements between reviewers in both phases were resolved by discussion until consensus.

\section{Data Extraction}

The information extracted from each study included the following: (1) study characteristics, including country and study period; (2) design and methodological characteristics, including type of study, sample size, drug of exposure, phenotypic outcomes, and how exposure and outcome were measured; and (3) genetic investigation considerations, including sample tissue, genetic approach, methodology of genetic investigation, genes and genetic variants investigated, and pharmacogenetic outcomes with risk estimates.

\section{RESULTS}

\section{General Characteristics of the Studies}

A total of 7,996 records were identified from the database searches, and the reference lists of relevant reviews and eligible studies (5,023 from PubMed, 2,947 from EMBASE, and 26 from the reference lists of relevant reviews; Figure 1). After removing duplicates, 7,320 studies were eligible for screening of the title and abstract. In the first screening, 7,204 studies were excluded and 116 publications were selected for assessment of the full text. The final list of eligible publications included 29 studies.

The general characteristics of the studies included in this review are presented in Table 1. The studies were published between 1999 and 2020 and were either cohort studies (16 studies) or case-control studies (13 studies). The sample sizes of the studies ranged from 20 to 9,774 individuals broken down as follows: $<100$ individuals (nine studies), 100-200 individuals (seven studies), 200-800 individuals (nine studies), and $>1,000$ individuals (four studies). Regarding the individuals, the studies examined neonates $(n=8,228)$, mothers $(n=14,481)$, and fathers $(n=105)$, as well as control individuals $(n=935$; not exposed to the drugs and unrelated to the cases) and genomic databases (four different databases used as control groups).

Several different drugs were investigated, including antidepressants (nine studies), thalidomide (seven studies), antiepileptic drugs (AEDs; five studies), glucocorticoids (four studies), acetaminophen (two studies), and sex hormones (estrogens, one study; 17-alpha hydroxyprogesterone caproate, one study). The main phenotypic outcomes evaluated by the studies were perinatal characteristics and complications (birth weight, Apgar scores, and respiratory distress), protein and neurotransmitter concentrations, congenital anomalies (heart, craniofacial, and limb anomalies, hypospadias, and clefts), and neurodevelopmental outcomes (including child mood, behavior, and executive functions, and disorders such as autism spectrum disorder [ASD]).

An overview of the research methodologies and results of the genetic analyses are presented in Table 2. One study performed whole-exome sequencing, whereas all other studies used a candidate gene approach. Various genotyping methods were applied in the gene candidate studies, and some studies utilized several different approaches. These methods included real-time polymerase chain reaction (real-time PCR; eleven studies), PCR (seven studies), PCR and restriction fragment length polymorphism (RFLP; six studies), genotyping by mass spectrometry (one study), microarray (two studies), Sanger sequencing (three studies), and targeted next-generation sequencing (two studies). DNA was extracted from whole blood (18 studies), cord blood (six studies), placental blood (one study), and/or saliva samples (13 studies) from mothers and/or children. Maternal genetics alone was evaluated in two studies, children's genetics in 14 studies, and both maternal and child genetics in 13 studies. In total, the studies found 48 polymorphisms in 29 genes, as well as several "likely gene-disruptive" mutations, associated with an increased risk of abnormal outcomes in children prenatally exposed to the drugs.

\section{Genetic Susceptibility Associated With Drug Teratogenicity}

The main outcomes regarding genetic teratology are presented below. The genes referred to in the following paragraphs are described with their full name and function in Appendix 4.

\section{Antidepressants}

Antidepressants, in particular serotonin reuptake inhibitors (SRIs) examined in eight out of nine studies, was the most investigated class of drug in the literature on genetic variations associated with adverse drug effects in utero. SLC6A4, which encodes the serotonin transporter, was a commonly investigated gene in studies on genetic vulnerability for a wide range of different antidepressants and phenotypic outcomes. Variants of SLC6A4 was associated with executive function accuracy 


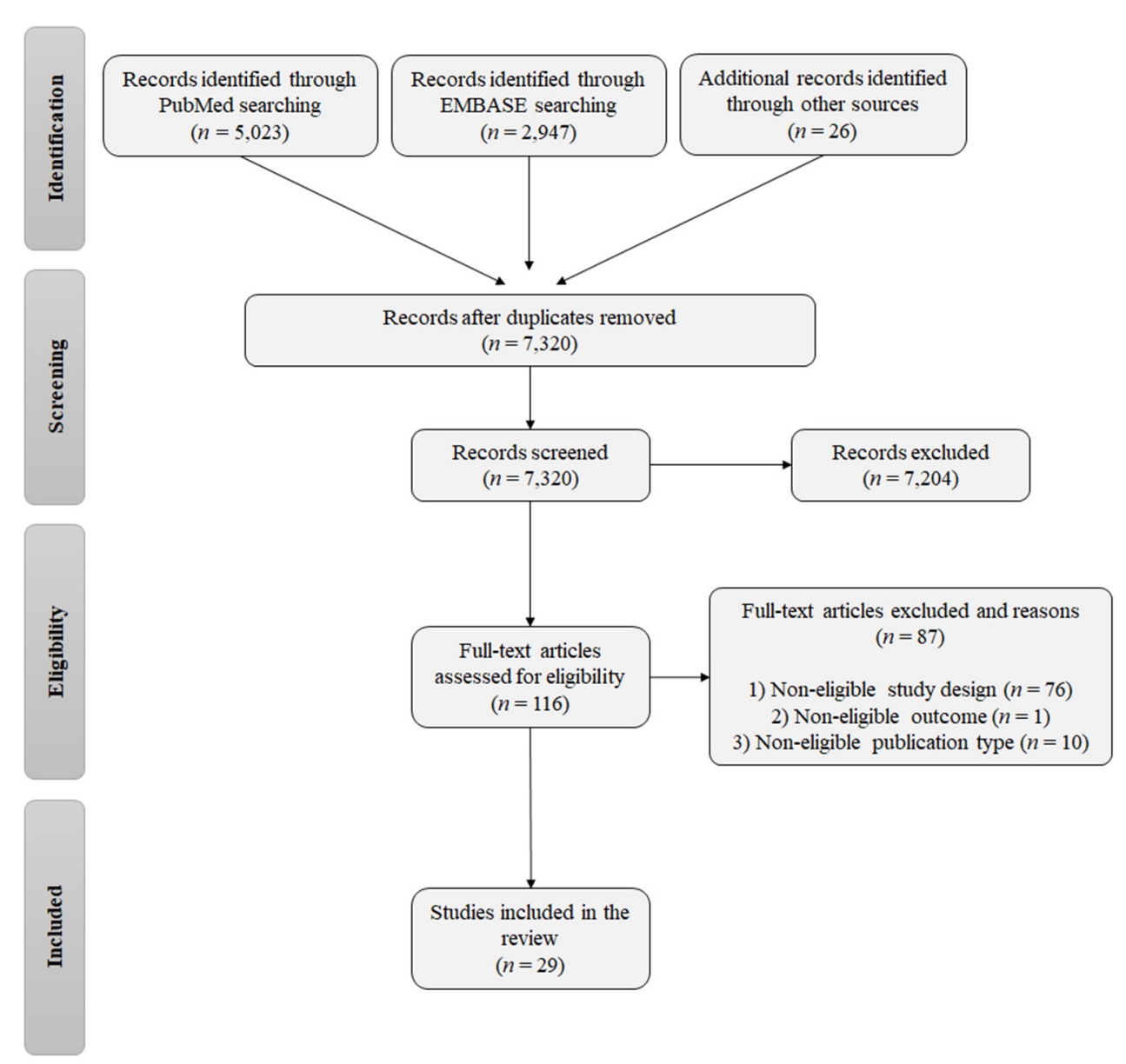

FIGURE 1 | Flowchart of the study screening and selection process.

in children (Weikum et al., 2013), blood reelin levels related to neonatal sleeping disturbances and irritability (Brummelte et al., 2013), and perinatal characteristics, such as birth weight, 5-min Apgar score, muscular tone, and respiratory complications (Oberlander et al., 2008). The MAO-A, COMT, and CYP2D6 genotypes were also associated with adverse outcomes upon antidepressant exposure, with genetic variants of these genes associated with serotonergic symptoms and abnormal neurotransmitter concentrations in infants (Hilli et al., 2009). Furthermore, genetic variants of SHMT1, BHMT, MGST1, MGMT, MTHFS, GNMT, and TRDMT1 were associated with an increased risk of congenital heart defects after prenatal antidepressant exposure (Nembhard et al., 2017). Finally, in individuals with ASD prenatally exposed to antidepressants, the presence of "likely gene-disruptive" mutations was associated with increased severity of symptoms of ASD (Ackerman et al., 2017).

\section{Thalidomide}

Thalidomide was the main individually investigated drug. All studies have been performed by the same research group in the approximately same sample of individuals (minor changes in sample sizes depending on the study). In these studies, the CRBN and NOS3, which are part of the CLR4-Cereblon complex, and act on angiogenic and oxidative stress processes, respectively, were reported to be associated with an increased risk of developing thalidomide embryopathy, a phenotypic spectrum of malformations characterized by limb reduction defects (Vargesson, 2009). The CRBN genetic variants rs1045433 and rs1620675 were associated with an increased risk of pre-axial limb anomalies and neurological anomalies, respectively (Vianna et al., 2016; Kowalski et al., 2020). In addition, NOS3 has deleterious variants (rs2070744, rs1799983, and rs61722009) associated with susceptibility to developing thalidomide embryopathy (Vianna et al., 2013; Kowalski et al., 2016).

\section{Antiepileptic Drugs}

MTHFR, associated with folate metabolism, was the most frequently investigated gene in studies on the genetic susceptibility to AED teratogenesis. The most commonly studied AEDs were valproic acid, carbamazepine, and phenytoin. Upon AED exposure, the maternal genetic variant rs1801133 of MTHFR was associated with an increased risk of major malformations and fetal anticonvulsant syndrome in the children 
TABLE 1 | Overview of the reviewed studies.

\begin{tabular}{|c|c|c|c|c|c|c|c|c|}
\hline & Reference & $\begin{array}{l}\text { Country; setting } \\
\text { (study period) }\end{array}$ & $\begin{array}{l}\text { Study } \\
\text { design }\end{array}$ & Sample size & $\begin{array}{l}\text { Drugs of } \\
\text { exposure }\end{array}$ & $\begin{array}{l}\text { Exposure } \\
\text { measures }\end{array}$ & $\begin{array}{l}\text { Phenotypic } \\
\text { outcomes }\end{array}$ & $\begin{array}{l}\text { Outcome } \\
\text { measures }\end{array}$ \\
\hline \multirow{6}{*}{ 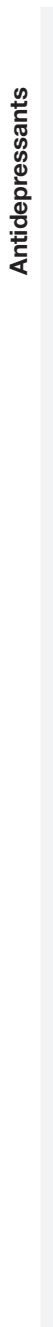 } & Corti et al. (2019) & Italy (2011-2016) & Case $\times$ control & $\begin{array}{l}\text { I SRls and maternal } \\
\text { depression/anxiety, } n=32 / 30 \text { : } \\
\text { Normal neonatal genotype, } n=10 \\
\text { Altered neonatal genotype, } n=22 \\
\text { Normal maternal genotype, } n=12 \\
\text { Altered maternal genotype, } n=1\end{array}$ & $\begin{array}{l}\text { Paroxetine, } \\
\text { venlafaxine, } \\
\text { sertraline, or } \\
\text { citalopram }\end{array}$ & $\begin{array}{l}\text { Medical records and } \\
\text { follow-up of drug } \\
\text { compliance during } \\
\text { pregnancy in monthly } \\
\text { obstetric and psychiatric } \\
\text { visits }\end{array}$ & $\begin{array}{l}\text { Preterm birth, neonatal } \\
\text { complications }^{\mathrm{a}} \text {, and } \\
\text { umbilical artery } \mathrm{pH} \text { and } \\
\text { base excess }\end{array}$ & $\begin{array}{l}\text { Medical records and follow-up by } \\
\text { multidisciplinary team at the hospital }\end{array}$ \\
\hline & $\begin{array}{l}\text { Ackerman et al. } \\
\text { (2017) }\end{array}$ & $\begin{array}{l}\text { USA; sample from the } \\
\text { Simons Simplex } \\
\text { Collection }\end{array}$ & Cohort & $\begin{array}{l}\text { Antidepressants and LGD } \\
\text { mutations } n=9 \\
\text { Antidepressants and no LGD } \\
\text { mutations } n=95 \\
\text { No antidepressants and LGD } \\
\text { mutations } n=335 \\
\text { No antidepressants and no LGD } \\
\text { mutations, } n=2,011 \text { (All } \\
\text { participants had } A S D \text { ) }\end{array}$ & $\begin{array}{l}\text { Amitriptyline, } \\
\text { bupropion, } \\
\text { citalopram, } \\
\text { escitalopram, } \\
\text { fluoxetine, } \\
\text { imipramine, } \\
\text { paroxetine, } \\
\text { sertraline, or } \\
\text { venlafaxine }\end{array}$ & $\begin{array}{l}\text { Parent interview; use of } \\
\text { antidepressants in } \geq 1 \\
\text { trimesters }\end{array}$ & ASD severity & $\begin{array}{l}\text { Clinician-measured (ADOS) or } \\
\text { parent-reported (ADI-R) ASD severity }\end{array}$ \\
\hline & Daud et al. (2017) & $\begin{array}{l}\text { The Netherlands; the } \\
\text { EUROCAT Northern } \\
\text { Netherlands database } \\
\text { (1997-2013) }\end{array}$ & Cohort & $\begin{array}{l}\text { SRIs and congenital heart anomaly, } \\
n=7 \text { mothers/children } \\
\text { No SRIs and congenital heart } \\
\text { anomaly, } n=28 \text { mothers/children }\end{array}$ & $\begin{array}{l}\text { Fluoxetine, } \\
\text { paroxetine, or } \\
\text { venlafaxine }\end{array}$ & $\begin{array}{l}\text { Medical records verified } \\
\text { by telephone interviews }\end{array}$ & $\begin{array}{l}\text { Congenital heart } \\
\text { anomalies }\end{array}$ & $\begin{array}{l}\text { Diagnoses of congenital heart anomalies } \\
\text { before } 10 \text { years of age based on ICD-9/10 } \\
\text { registered in the EUROCAT Northern } \\
\text { Netherlands database }\end{array}$ \\
\hline & $\begin{array}{l}\text { Nembhard et al. } \\
\text { (2017) }\end{array}$ & $\begin{array}{l}\text { USA; the US National } \\
\text { Birth Defects } \\
\text { Prevention Study } \\
\text { (NBDPS; 1997-2008) }\end{array}$ & Case $\times$ control & $\begin{array}{l}\text { I SSRIs and congenital heart } \\
\text { anomaly, } n=57 \text { mothers/children } \\
\text { SSRIs and no congenital heart } \\
\text { anomaly, } n=54 \text { mothers/children } \\
\text { No SSRIs and congenital heart } \\
\text { anomaly, } n=1,119 \\
\text { mothers/children } \\
\text { No SSRIs and no congenital heart } \\
\text { anomaly, } n=1,590 \\
\text { mothers/children }\end{array}$ & $\begin{array}{l}\text { Sertraline, fluoxetine, } \\
\text { paroxetine, } \\
\text { citalopram, or } \\
\text { escitalopram }\end{array}$ & $\begin{array}{l}\text { Maternal interview of } \\
\text { consecutive drug use for } \\
\text { at least } 2 \text { months } \\
\text { between } 2 \text { months } \\
\text { before conception and } 3 \\
\text { months after conception }\end{array}$ & $\begin{array}{l}\text { Congenital heart } \\
\text { ranomalies }\end{array}$ & $\begin{array}{l}\text { Diagnoses of congenital heart anomalies } \\
\text { based on the classification system } \\
\text { specifically developed for the National Birth } \\
\text { Defects Prevention Study, incorporating } \\
\text { cardiac phenotype, complexity of the } \\
\text { phenotype, and extra-cardiac anomalies }\end{array}$ \\
\hline & $\begin{array}{l}\text { Weikum et al. } \\
\text { (2013) }\end{array}$ & $\begin{array}{l}\text { Canada; part of a study } \\
\text { on the effects of } \\
\text { antenatal SSRI } \\
\text { exposure }\end{array}$ & Cohort & $\begin{array}{l}\text { SRls and maternal mood disorder, } n \\
=26 \text { children } \\
\text { No SRIs and maternal mood } \\
\text { symptoms, } n=38 \text { children }\end{array}$ & $\begin{array}{l}\text { Paroxetine, } \\
\text { fluoxetine, sertraline, } \\
\text { venlafaxine, or } \\
\text { citalopram }\end{array}$ & $\begin{array}{l}\text { Physician- or } \\
\text { self-reported }\end{array}$ & $\begin{array}{l}\text { Child mood, behavior, } \\
\text { and executive functions }\end{array}$ & $\begin{array}{l}\text { Child mood and behavior assessed with } \\
\text { the mental health symptomatology section } \\
\text { of the MacArthur Health and Behavior } \\
\text { Questionnaire. Executive functions } \\
\text { assessed by the Hearts and Flowers task }\end{array}$ \\
\hline & $\begin{array}{l}\text { Brummelte et al. } \\
\text { (2013) }\end{array}$ & Canada (2006-2009) & Cohort & $\begin{array}{l}\text { SRIs, } n=17 \text { mothers/children } \\
\text { No SRIs, } n=28 / 26 \\
\text { mothers/children }\end{array}$ & $\begin{array}{l}\text { Paroxetine, } \\
\text { fluoxetine, sertraline, } \\
\text { venlafaxine, } \\
\text { citalopram, or } \\
\text { escitalopram }\end{array}$ & $\begin{array}{l}\text { Medical records on } \\
\text { medication, dose, and } \\
\text { indication }\end{array}$ & $\begin{array}{l}\text { Levels of reelin in cord } \\
\text { blood and maternal } \\
\text { blood }\end{array}$ & $\begin{array}{l}\text { Western blot analysis of protein levels in } \\
\text { maternal and umbilical cord serum }\end{array}$ \\
\hline
\end{tabular}




\begin{tabular}{|c|c|c|c|c|c|c|c|c|}
\hline & Reference & $\begin{array}{l}\text { Country; setting } \\
\text { (study period) }\end{array}$ & $\begin{array}{l}\text { Study } \\
\text { design }\end{array}$ & Sample size & $\begin{array}{l}\text { Drugs of } \\
\text { exposure }\end{array}$ & $\begin{array}{l}\text { Exposure } \\
\text { measures }\end{array}$ & $\begin{array}{l}\text { Phenotypic } \\
\text { outcomes }\end{array}$ & $\begin{array}{l}\text { Outcome } \\
\text { measures }\end{array}$ \\
\hline & $\begin{array}{l}\text { Oberlander et al. } \\
\text { (2010) }\end{array}$ & $\begin{array}{l}\text { Canada; part of a study } \\
\text { on the effects of } \\
\text { antenatal SSRI } \\
\text { exposure }\end{array}$ & Cohort & $\begin{array}{l}\text { SRIs and maternal mood disorder, } n \\
=33 \text { children } \\
\text { No SRIs and maternal mood } \\
\text { symptoms, } n=42 \text { children }\end{array}$ & $\begin{array}{l}\text { Paroxetine, } \\
\text { fluoxetine, sertraline, } \\
\text { venlafaxine, or } \\
\text { citalopram }\end{array}$ & $\begin{array}{l}\text { Physician- or } \\
\text { self-reported }\end{array}$ & Child behavior & $\begin{array}{l}\text { Child behavior was evaluated with the } \\
\text { Child Behavior Checklist at } 3 \text { years of age }\end{array}$ \\
\hline & Hilli et al. (2009) & $\begin{array}{l}\text { Finland; part of a } \\
\text { controlled prospective } \\
\text { study by Laine et al., } \\
2003 \text { (1997-2000) }\end{array}$ & Cohort & SSRIs, $n=20$ children & $\begin{array}{l}\text { Citalopram or } \\
\text { fluoxetine }\end{array}$ & $\begin{array}{l}\text { Medical records on the } \\
\text { medication, dose, and } \\
\text { indication }\end{array}$ & $\begin{array}{l}\text { Perinatal serotonergic } \\
\text { symptoms and } \\
\text { neurotransmitter blood } \\
\text { concentrations }\end{array}$ & $\begin{array}{l}\text { Assessment of serotonergic symptoms by } \\
\text { pediatricians (Sternbach, 1991). } \\
\text { Neurotransmitter concentrations } \\
\text { measured from whole blood and umbilical } \\
\text { vein blood samples }\end{array}$ \\
\hline & $\begin{array}{l}\text { Oberlander et al. } \\
\text { (2008) }\end{array}$ & $\begin{array}{l}\text { Canada; part of a study } \\
\text { on the effects of } \\
\text { antenatal SSRI } \\
\text { exposure }\end{array}$ & Cohort & $\begin{array}{l}\text { SRIs, } n=37 \text { mothers/children } \\
\text { No SRIs, } n=47 \text { mothers/children }\end{array}$ & $\begin{array}{l}\text { Paroxetine, } \\
\text { fluoxetine, sertraline, } \\
\text { venlafaxine, or } \\
\text { citalopram }\end{array}$ & $\begin{array}{l}\text { Medical records on } \\
\text { medication and } \\
\text { indication }\end{array}$ & $\begin{array}{l}\text { Preterm birth and } \\
\text { adverse neonatal } \\
\text { outcomes (e.g., birth } \\
\text { weight, Apgar score, } \\
\text { muscle tone, respiratory } \\
\text { distress, and jitteriness) }\end{array}$ & $\begin{array}{l}\text { Medical records and follow-up at the } \\
\text { hospital }\end{array}$ \\
\hline \multirow{4}{*}{ 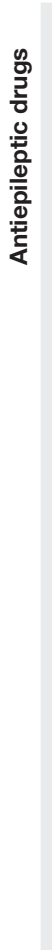 } & Jose et al. (2014) & $\begin{array}{l}\text { India; part of the Kerala } \\
\text { Registry of Epilepsy } \\
\text { and Pregnancy (KREP) }\end{array}$ & $\begin{array}{r}\text { Case } \times \text { control } \mathrm{A} \\
\mathrm{c} \\
\mathrm{n} \\
\mathrm{A} \\
\mathrm{c} \\
\mathrm{a}\end{array}$ & $\begin{array}{l}\text { AEDs, maternal epilepsy and } \\
\text { children with congenital anomalies, } \\
n=143 \text { mothers } \\
\text { AEDs, maternal epilepsy and } \\
\text { children without congenital } \\
\text { anomalies, } n=123 \text { mothers }\end{array}$ & $\begin{array}{l}\text { Carbamazepine, } \\
\text { valproic acid, } \\
\text { phenytoin, } \\
\text { clonazepam, or } \\
\text { phenobarbital }\end{array}$ & $\begin{array}{l}\text { Medical records of the } \\
\text { registry }\end{array}$ & $\begin{array}{l}\text { Congenital anomalies } \\
\text { (main focus on } \\
\text { cardio-vascular } \\
\text { anomalies) }\end{array}$ & $\begin{array}{l}\text { Clinical examination of malformations at } \\
\text { birth. Echocardiography and abdomen } \\
\text { ultrasonography at } 3 \text { months of age }\end{array}$ \\
\hline & $\begin{array}{l}\text { Azzato et al. } \\
(2010)\end{array}$ & $\begin{array}{l}\text { USA; part of the } \\
\text { Collaborative Perinatal } \\
\text { Project (CPP) database } \\
\text { (1959-1966) }\end{array}$ & Cohort & $\begin{array}{l}\text { AEDs, } n=155 / 174 \\
\text { (mothers/children) }\end{array}$ & Phenytoin & $\begin{array}{l}\text { Questionnaire data and } \\
\text { maternal medical } \\
\text { records }\end{array}$ & Craniofacial anomalies & $\begin{array}{l}\text { Craniofacial anomalies considered if } \\
\text { microcephaly, abnormal sutures, midfacial } \\
\text { hypoplasia, cleft lip, palate or gum, or } \\
\text { deformed/low-set ears were noted in } \\
\text { medical records. A standardized } \\
\text { evaluation form assessing the presence of } \\
\text { anomalies was subsequently completed } \\
\text { by physicians }\end{array}$ \\
\hline & Dean et al. (2007) & Scotland (1976-2002) & Case $\times$ control $A$ & $\begin{array}{l}\text { AEDs, } n=276 / 172 / 86 \\
\text { (children/mothers/fathers) } \\
\text { No AEDs, } n=646 \text { healthy blood } \\
\text { donors }\end{array}$ & $\begin{array}{l}\text { Valproic acid, } \\
\text { phenobarbitone, } \\
\text { carbamazepine, } \\
\text { phenytoin, } \\
\text { lamotrigine, or } \\
\text { polytherapy }\end{array}$ & $\begin{array}{l}\text { Medical records and } \\
\text { maternal interviews on } \\
\text { AED use }\end{array}$ & $\begin{array}{l}\text { Fetal anticonvulsant } \\
\text { syndrome, major } \\
\text { malformations, and } \\
\text { neurodevelopmental } \\
\text { disorders }\end{array}$ & $\begin{array}{l}\text { Facial photographs reviewed by a } \\
\text { physician for fetal anticonvulsant } \\
\text { syndrome (diagnosed if } \geq 1 \text { major and } \geq 1 \\
\text { minor feature appeared in the child, or if } \\
\text { (s)he had } \geq 3 \text { minor features). Children } \\
\text { without fetal anticonvulsant syndrome } \\
\text { were included in analyses of major } \\
\text { malformations and neurodevelopmental } \\
\text { disorders }\end{array}$ \\
\hline & Kini et al. (2007) & $\begin{array}{l}\text { United Kingdom } \\
\text { (2000-2004) }\end{array}$ & Cohort & $\begin{array}{l}\text { AEDs and epilepsy, } n=153 \\
\text { mothers/children } \\
\text { No AEDs and no epilepsy, } n=236 \\
\text { mothers/children }\end{array}$ & $\begin{array}{l}\text { Valproic acid, } \\
\text { carbamazepine, } \\
\text { another } \\
\text { monotherapy, or } \\
\text { polytherapy }\end{array}$ & $\begin{array}{l}\text { Medical records on AED } \\
\text { use and dose }\end{array}$ & Major malformations & $\begin{array}{l}\text { Major malformations defined as structural } \\
\text { abnormalities requiring medical or surgical } \\
\text { intervention to prevent disability using the } \\
\text { EUROCAT list of congenital malformations } \\
\text { as a guideline }\end{array}$ \\
\hline
\end{tabular}

Project (CPP) database

maternal medica

records

(Continued) 
TABLE 1 | Continued

\begin{tabular}{|c|c|c|c|c|c|c|c|c|}
\hline & Reference & $\begin{array}{l}\text { Country; setting } \\
\text { (study period) }\end{array}$ & $\begin{array}{l}\text { Study } \\
\text { design }\end{array}$ & Sample size & $\begin{array}{l}\text { Drugs of } \\
\text { exposure }\end{array}$ & $\begin{array}{l}\text { Exposure } \\
\text { measures }\end{array}$ & $\begin{array}{l}\text { Phenotypic } \\
\text { outcomes }\end{array}$ & $\begin{array}{l}\text { Outcome } \\
\text { measures }\end{array}$ \\
\hline & Dean et al. (1999) & Scotland & Case $\times$ control & $\begin{array}{l}\text { I AEDs, } n=46 / 36 / 19 \\
\text { (children/mothers/fathers) } \\
\text { No AEDs, } n=152 \text { adults matched } \\
\text { to the parents }\end{array}$ & $\begin{array}{l}\text { Valproic acid, } \\
\text { carbamazepine, and } \\
\text { phenytoin }\end{array}$ & $\begin{array}{l}\text { The Fetal Anticonvulsant } \\
\text { Syndrome Association } \\
\text { registry }\end{array}$ & $\begin{array}{l}\text { Fetal anticonvulsant } \\
\text { syndrome }\end{array}$ & $\begin{array}{l}\text { Fetal anticonvulsant syndrome in the } \\
\text { presence of a characteristic facial } \\
\text { appearance and at least one compatible } \\
\text { clinical finding (anticonvulsant-associated } \\
\text { malformation, medical disorder, or } \\
\text { developmental delay), and no other } \\
\text { identifiable etiology }\end{array}$ \\
\hline \multirow[t]{4}{*}{ 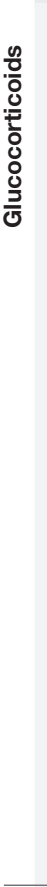 } & $\begin{array}{l}\text { Van Der Voorn } \\
\text { et al. (2015) }\end{array}$ & $\begin{array}{l}\text { The Netherlands; part } \\
\text { of the Project On } \\
\text { Preterm and Small for } \\
\text { gestational age infants } \\
\text { (POPS) cohort (1983) }\end{array}$ & Cohort & $\begin{array}{l}\text { Betamethasone, } n=71 \text { children } \\
\text { No betamethasone, } n=273 \\
\text { children }\end{array}$ & Betamethasone & Medical records & $\begin{array}{l}\text { Behavior and } \\
\text { intelligence }\end{array}$ & $\begin{array}{l}\text { Behavior assessed by the YASR } \\
\text { (self-reported) and YABCL } \\
\text { (parent-reported) questionnaires. } \\
\text { Intellectual functioning examined with the } \\
\text { Multicultural Capacity Test-Intermediate } \\
\text { Level (Bleichrodt and van den Berg, 2000) }\end{array}$ \\
\hline & Haas et al. (2013) & $\begin{array}{l}\text { USA; part of the } \\
\text { betamethasone (BMZ) } \\
\text { pharmacogenetics } \\
\text { cohort }\end{array}$ & Cohort & $\begin{array}{l}\text { Betamethasone, } n=109 / 117 \\
\text { (mothers/children) }\end{array}$ & Betamethasone & Medical records & $\begin{array}{l}\text { Adverse neonatal } \\
\text { respiratory outcomes }\end{array}$ & $\begin{array}{l}\text { Neonates with respiratory difficulties } \\
\text { included } \\
\text { - Neonates needing respiratory support } \\
\text { or surfactants (data extracted from } \\
\text { neonatal charts) } \\
\text { - Neonates diagnosed with } \\
\text { bronchopulmonary dysplasia by } \\
\text { pediatricians (standard criteria from the } \\
\text { Neonatal Research Network of the } \\
\text { National Institute of Child Health and } \\
\text { Human Development) }\end{array}$ \\
\hline & Oretti et al. (2009) & Italy (2005-2006) & Cohort & $\begin{array}{l}\text { Betamethasone, } n=62 \text { preterm } \\
\text { neonates }\end{array}$ & Betamethasone & Medical records & $\begin{array}{l}\text { Respiratory distress } \\
\text { syndrome }\end{array}$ & $\begin{array}{l}\text { Diagnosis of respiratory distress syndrome } \\
\text { based on clinical and radiographic criteria } \\
\text { (tachypnea, chest retractions, cyanosis in } \\
\text { room air persisting } 48-96 \mathrm{~h} \text { and typical } \\
\text { chest X-ray) }\end{array}$ \\
\hline & $\begin{array}{l}\text { Bertalan et al. } \\
\text { (2008) }\end{array}$ & $\begin{array}{l}\text { Hungary; part of a } \\
\text { neonate cohort at } \\
\text { Semmelweis University }\end{array}$ & Cohort & $\begin{array}{l}\text { Dexamethasone, } n=57 \text { preterm } \\
\text { neonates; } \\
\text { No dexamethasone, } n=68 \\
\text { preterm neonates }\end{array}$ & Dexamethasone & Medical records & $\begin{array}{l}\text { Birth weight and } \\
\text { perinatal complications }{ }^{c}\end{array}$ & $\begin{array}{l}\text { Medical charts and diagnoses based on } \\
\text { clinical and radiographic observations and } \text { tests }^{d}\end{array}$ \\
\hline 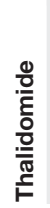 & $\begin{array}{l}\text { Kowalski et al. } \\
\text { (2020) }\end{array}$ & Brazil & Case $\times$ control & $\begin{array}{l}\text { I Thalidomide, } n=27 \text { affected } \\
\text { individuals (non-Finnish European } \\
\text { population of ExAC, 1,000 } \\
\text { Genomes, and ABraOM databases } \\
\text { were used as a control group for } \\
\text { genetic comparisons) }\end{array}$ & Thalidomide & $\begin{array}{l}\text { Medication use defined } \\
\text { based on neonatal } \\
\text { phenotype }\end{array}$ & $\begin{array}{l}\text { Thalidomide } \\
\text { embryopathy and their } \\
\text { specific phenotypes }\end{array}$ & $\begin{array}{l}\text { The authors characterized congenital } \\
\text { anomalies related to thalidomide exposure } \\
\text { according to a guideline they created. } \\
\text { Congenital anomalies were consistent with } \\
\text { typical thalidomide embryopathy }\end{array}$ \\
\hline
\end{tabular}


TABLE 1 | Continued

\begin{tabular}{|c|c|c|c|c|c|c|c|}
\hline Reference & $\begin{array}{l}\text { Country; setting } \\
\text { (study period) }\end{array}$ & $\begin{array}{l}\text { Study } \\
\text { design }\end{array}$ & Sample size & $\begin{array}{l}\text { Drugs of } \\
\text { exposure }\end{array}$ & $\begin{array}{l}\text { Exposure } \\
\text { measures }\end{array}$ & $\begin{array}{l}\text { Phenotypic } \\
\text { outcomes }\end{array}$ & $\begin{array}{l}\text { Outcome } \\
\text { measures }\end{array}$ \\
\hline $\begin{array}{l}\text { Gomes et al. } \\
\text { (2019) }\end{array}$ & Brazil & Case $\times$ control & $\begin{array}{l}\text { Thalidomide, } n=36 \text { affected } \\
\text { individuals; } \\
\text { Assumption of no thalidomide by } \\
\text { absence of congenital anomalies } \\
\text { and thalidomide embryopathy, } n= \\
135 \text { individuals }\end{array}$ & Thalidomide & $\begin{array}{l}\text { Medication use defined } \\
\text { based on neonatal } \\
\text { phenotype }\end{array}$ & $\begin{array}{l}\text { Thalidomide } \\
\text { embryopathy and } \\
\text { embryopathy-specific } \\
\text { phenotypes }\end{array}$ & $\begin{array}{l}\text { The authors characterized congenital } \\
\text { anomalies related to thalidomide exposure } \\
\text { according to a guideline they created. } \\
\text { Congenital anomalies were consistent with } \\
\text { typical thalidomide embryopathy }\end{array}$ \\
\hline $\begin{array}{l}\text { Gomes et al. } \\
\text { (2018) }\end{array}$ & Brazil & Case $\times$ control & $\begin{array}{l}\text { Thalidomide, } n=35 \text { affected } \\
\text { individuals (non-Finnish European } \\
\text { population of gnomAD database } \\
\text { was used as a control group) }\end{array}$ & Thalidomide & $\begin{array}{l}\text { Medication use defined } \\
\text { based on neonatal } \\
\text { phenotype }\end{array}$ & $\begin{array}{l}\text { Thalidomide } \\
\text { embryopathy and } \\
\text { embryopathy-specific } \\
\text { phenotypes }\end{array}$ & $\begin{array}{l}\text { The authors characterized congenital } \\
\text { anomalies related to thalidomide exposure } \\
\text { according to a guideline they created. } \\
\text { Congenital anomalies were consistent with } \\
\text { typical thalidomide embryopathy }\end{array}$ \\
\hline $\begin{array}{l}\text { Kowalski et al. } \\
\text { (2017) }\end{array}$ & Brazil & Case $\times$ control & $\begin{array}{l}\text { Thalidomide, } n=36 \text { affected } \\
\text { individuals } \\
\text { Assumption of no thalidomide by } \\
\text { absence of congenital anomalies } \\
\text { and thalidomide embryopathy, } n= \\
136 \text { individuals }\end{array}$ & Thalidomide & $\begin{array}{l}\text { Medication use defined } \\
\text { based on neonatal } \\
\text { phenotype }\end{array}$ & $\begin{array}{l}\text { Thalidomide } \\
\text { embryopathy and } \\
\text { embryopathy-specific } \\
\text { phenotypes }\end{array}$ & $\begin{array}{l}\text { The authors characterized congenital } \\
\text { anomalies related to thalidomide exposure } \\
\text { according to a guideline they created. } \\
\text { Congenital anomalies were consistent with } \\
\text { typical thalidomide embryopathy }\end{array}$ \\
\hline $\begin{array}{l}\text { Kowalski et al. } \\
\text { (2016) }\end{array}$ & Brazil & Case $\times$ control & $\begin{array}{l}\text { Thalidomide, } n=38 \text { affected } \\
\text { individuals } \\
\text { Assumption of no thalidomide by } \\
\text { absence of congenital anomalies } \\
\text { and thalidomide embryopathy, } n= \\
137 \text { individuals }\end{array}$ & Thalidomide & $\begin{array}{l}\text { Medication use defined } \\
\text { based on neonatal } \\
\text { phenotype }\end{array}$ & $\begin{array}{l}\text { Thalidomide } \\
\text { embryopathy and } \\
\text { embryopathy-specific } \\
\text { phenotypes }\end{array}$ & $\begin{array}{l}\text { The authors characterized congenital } \\
\text { anomalies related to thalidomide exposure } \\
\text { according to a guideline they created. } \\
\text { Congenital anomalies were consistent with } \\
\text { typical thalidomide embryopathy }\end{array}$ \\
\hline $\begin{array}{l}\text { Vianna et al. } \\
\text { (2016) }\end{array}$ & Brazil & Case $\times$ control & $\begin{array}{l}\text { Thalidomide, } n=38 \text { affected } \\
\text { individuals } \\
\text { Assumption of no thalidomide by } \\
\text { absence of congenital anomalies } \\
\text { and thalidomide embryopathy, } n= \\
136 \text { individuals }\end{array}$ & Thalidomide & $\begin{array}{l}\text { Medication use defined } \\
\text { based on neonatal } \\
\text { phenotype }\end{array}$ & $\begin{array}{l}\text { Thalidomide } \\
\text { embryopathy and } \\
\text { embryopathy-specific } \\
\text { phenotypes }\end{array}$ & $\begin{array}{l}\text { The authors characterized congenital } \\
\text { anomalies related to thalidomide exposure } \\
\text { according to a guideline they created. } \\
\text { Congenital anomalies were consistent with } \\
\text { typical thalidomide embryopathy }\end{array}$ \\
\hline $\begin{array}{l}\text { Vianna et al. } \\
\text { (2013) }\end{array}$ & Brazil & Case $\times$ control & $\begin{array}{l}\text { Thalidomide, } n=28 / 27 \text { (affected } \\
\text { individuals/their relatives) } \\
\text { Assumption of no thalidomide by } \\
\text { absence of congenital anomalies } \\
\text { and thalidomide embryopathy, } n= \\
137 \text { individuals }\end{array}$ & Thalidomide & $\begin{array}{l}\text { Medication use defined } \\
\text { based on neonatal } \\
\text { phenotype }\end{array}$ & $\begin{array}{l}\text { Thalidomide } \\
\text { embryopathy and } \\
\text { embryopathy-specific } \\
\text { phenotypes }\end{array}$ & $\begin{array}{l}\text { The authors characterized congenital } \\
\text { anomalies related to thalidomide exposure } \\
\text { according to a guideline they created. } \\
\text { Congenital anomalies were consistent with } \\
\text { typical thalidomide embryopathy }\end{array}$ \\
\hline
\end{tabular}


TABLE 1 | Continued

\begin{tabular}{|c|c|c|c|c|c|c|c|c|}
\hline & Reference & $\begin{array}{l}\text { Country; setting } \\
\text { (study period) }\end{array}$ & $\begin{array}{l}\text { Study } \\
\text { design }\end{array}$ & Sample size & $\begin{array}{l}\text { Drugs of } \\
\text { exposure }\end{array}$ & $\begin{array}{l}\text { Exposure } \\
\text { measures }\end{array}$ & $\begin{array}{l}\text { Phenotypic } \\
\text { outcomes }\end{array}$ & $\begin{array}{l}\text { Outcome } \\
\text { measures }\end{array}$ \\
\hline \multirow[t]{4}{*}{ 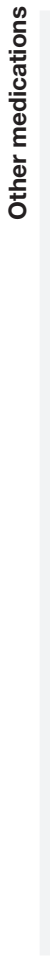 } & $\begin{array}{l}\text { Bustos et al. } \\
(2017)\end{array}$ & $\begin{array}{l}\text { USA; part of a trial on } \\
\text { omega- } 3 \text { use and } \\
\text { spontaneous preterm } \\
\text { births }\end{array}$ & Cohort & $\begin{array}{l}\text { 17-alpha hydroxyprogesterone } \\
\text { caproate, } n=268 \text { mothers }\end{array}$ & $\begin{array}{l}\text { 17-alpha hydroxy- } \\
\text { progesterone } \\
\text { caproate }\end{array}$ & Medical records & $\begin{array}{l}\text { 17-alpha } \\
\text { hydroxy-progesterone } \\
\text { caproate plasma } \\
\text { concentrations and } \\
\text { spontaneous preterm } \\
\text { births }\end{array}$ & $\begin{array}{l}\text { 17-alpha hydroxyprogesterone caproate } \\
\text { plasma concentrations determined by } \\
\text { high-performance liquid } \\
\text { chromatography-mass spectrometry (limit } \\
\text { of detection: } 1 \mathrm{ng} / \mathrm{mL} \text { ). Preterm birth } \\
\text { based on the gestational age at delivery }\end{array}$ \\
\hline & $\begin{array}{l}\text { Van Der Zanden } \\
\text { et al. (2012) }\end{array}$ & $\begin{array}{l}\text { The Netherlands; } \\
\text { subset of the AGORA } \\
\text { biobank at Radboud } \\
\text { University Nijmegen } \\
\text { Medical Center } \\
\text { (1980-2008) }\end{array}$ & Cohort & $\begin{array}{l}\text { Estrogens and hypospadias, } n=29 \\
\text { children } \\
\text { No estrogens and hypospadias, } n \\
=580 \text { children }\end{array}$ & Estrogens & Questionnaires & Hypospadias & $\begin{array}{l}\text { Medical records (no information on criteria } \\
\text { for diagnosing syndromic hypospadias) }\end{array}$ \\
\hline & $\begin{array}{l}\text { Perzanowski et al. } \\
\text { (2010) }\end{array}$ & USA (1998-2006) & Cohort & $\begin{array}{l}\text { Acetaminophen, } n=103 \\
\text { mothers/children } \\
\text { No acetaminophen, } n=198 \\
\text { mothers/children }\end{array}$ & Acetaminophen & Questionnaires & $\begin{array}{l}\text { Wheeze at } 1-3 \text { and } 5 \\
\text { years, and seroatopy }\end{array}$ & $\begin{array}{l}\text { Wheeze at } 1,2 \text {, or } 3 \text { years if } \geq 1 \text { episode } \\
\text { was reported in } \geq 1 \text { interview during these } \\
\text { years. Wheeze at } 5 \text { years if an episode } \\
\text { was reported in the previous } 12 \text { months } \\
\text { according to the ISAAC questionnaire. } \\
\text { Seroatopy if IgE antibodies in serum } \\
\geq 0.35 \mathrm{IU} / \mathrm{mL} \text { against } D \text {. farinae, mouse, } \\
\text { cockroach, cat, or dog }\end{array}$ \\
\hline & $\begin{array}{l}\text { Shaheen et al. } \\
\text { (2010) }\end{array}$ & $\begin{array}{l}\text { Unite Kingdom; Avon } \\
\text { Longitudinal Study of } \\
\text { Parents and Children } \\
\text { (ALSPAC; 1991-1992) }\end{array}$ & Cohort & $\begin{array}{l}\text { Acetaminophen in early pregnancy } \\
(<18-20 \text { weeks), } n=4,952 \\
\text { mothers/children } \\
\text { Acetaminophen in late pregnancy } \\
\text { (20-32 weeks), } n=4,822 \\
\text { mothers/children }\end{array}$ & Acetaminophen & Questionnaires & $\begin{array}{l}\text { Wheezing, asthma, } \\
\text { eczema, hay fever, } \\
\text { atopy, and lung function } \\
\text { at } 6.5-8.5 \text { years }\end{array}$ & $\begin{array}{l}\text { Children were defined as having wheezing, } \\
\text { eczema, and hay fever if mothers } \\
\text { responded positively to the question in the } \\
\text { interview; asthma was considered based } \\
\text { on diagnosis; atopy was defined as a } \\
\text { positive reaction to } D \text {. pteronyssinus, cat, } \\
\text { or grass; lung function was measured by } \\
\text { spirometry }\end{array}$ \\
\hline
\end{tabular}

a Low birth weight (<2,500 g), small for gestational age, APGAR scores (1 and $5 \mathrm{~min})<7$, neonatal breathing problems, neurological symptoms, respiratory distress syndrome, need of access to neonatal intensive care unit, persistent pulmonary hypertension of the neonate, poor neonatal adaptation syndrome; neonatal abstinence syndrome and transient tachypnea of the neonate. ${ }^{b}$ Single heart anomalies, part of complex heart anomalies (including cardiovascular anomalies) or part of complex anomalies involving other organ systems. Diagnosis codes from ICD-9 included 745-746, 7,470-7,474 (excluding 74,550, persistent foramen ovale), and from ICD-10 included Q20-Q26 (excluding Q2111, persistent foramen ovale). These encompass common arterial truncus, transposition of great vessels, single ventricle, ventricular/atrial/atrioventricular septal defects, tetralogy of Fallot, tricuspid atresia and stenosis, Ebstein's anomaly, pulmonary valve stenosis, pulmonary valve atresia, aortic valve atresia/stenosis, hypoplastic left/right heart syndrome, coarctation of aorta, total anomalous pulmonary venous return and patent ductus arteriosus. ${ }^{\circ}$ Perinatal complications included: necrotizing enterocolitis, intraventricular hemorrhage, patent ductus arteriosus, respiratory distress syndrome, bronchopulmonary dysplasia, and sepsis. ${ }^{d}$ The disorders were diagnosed as follows: necrotizing enterocolitis: clinical and radiological symptoms; intraventricular hemorrhage: neurosonograms; patent ductus arteriosus: echocardiography and clinical signs 5 days after birth; respiratory distress syndrome: need for respiratory support and oxygen upon presence of radiographic chest findings; bronchopulmonary dysplasia: oxygen dependency from 32 weeks of gestational age onwards; sepsis: clinical evidence and/or positive blood/cerebrospinal fluid cultures. ${ }^{~}$ ExAC database, EXAC data is now available in the gnomAD browser; 1,000 Genomes, around 3,000 genomes from individuals of different populations; ABraOM database, 609 exomes from Brazilian individuals. ${ }^{f}$ gnomAD database, 125,748 exomes and 15,708 whole genomes from individuals of different populations. ADI-R, Autism Diagnostic Interview-revised; ADOS, autism diagnostic observation schedule; AED, anti-epileptic drug; ASD, autism spectrum disorder; ICD-9/10, International Statistical Classification of Diseases and Related Health Problems 9th/10th Revision; LGD, likely gene-disrupting; SSRI, selective serotonin reuptake inhibitor; SRI, serotonin reuptake inhibitor; YABCL, Young Adult Behavio Checklist; YASR, Young Adult Self Report; TE, thalidomide embryopathy; ISAAC, International Study of Asthma and Allergies in Childhood. 
TABLE 2 | Overview of pharmacogenetic investigations and results.

\begin{tabular}{|c|c|c|c|c|c|c|c|}
\hline & Reference & Sample tissue & Approach & Method & Gene(s) investigated & Genetic variants investigated & Pharmacogenetic outcomes \\
\hline \multirow[t]{4}{*}{ 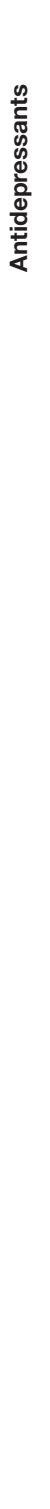 } & Corti et al. (2019) & $\begin{array}{l}\text { Maternal blood and } \\
\text { neonatal cord blood } \\
\text { samples at birth }\end{array}$ & Candidate genes & Real-time PCR & $\begin{array}{l}\text { CYP2D6, CYP2C19, } \\
\text { CYP2B6, CYP3A4, and } \\
\text { CYP3A5 }\end{array}$ & $\begin{array}{l}\text { CYP2D6: CYP2D6*3, } \\
\text { CYP2D6*4, CYP2D6*5, } \\
\text { CYP2D6*6, rs1080985 and gene } \\
\text { duplication; CYP2C19: } \\
\text { CYP2C19*2, CYP2C19*3, and } \\
\text { CYP2C19*17; CYP2B6: } \\
\text { CYP2B6*6; CYP3A4: } \\
\text { CYP3A4*22; CYP3A5: } \\
\text { CYP3A5*3 }\end{array}$ & $\begin{array}{l}\text { - No interaction between the investigated genes } \\
\text { and antidepressant exposure on the phenotypic } \\
\text { outcomes }(p>0.05)\end{array}$ \\
\hline & $\begin{array}{l}\text { Ackerman et al. } \\
\text { (2017) }\end{array}$ & $\begin{array}{l}\text { Neonatal blood } \\
\text { samples }\end{array}$ & Whole-exome & $\begin{array}{l}\text { Whole-exome } \\
\text { sequencing }\end{array}$ & $\begin{array}{l}\text { Genes exhibiting de } \\
\text { novo LGD mutations } \\
\text { [4,234 genes with de } \\
\text { novo mutations } \\
\text { identified in lossifov } \\
\text { et al. (2014)] }\end{array}$ & $\begin{array}{l}\text { De novo LGD mutations } \\
\text { (nonsense, splice site, and } \\
\text { frame-shift) }\end{array}$ & $\begin{array}{l}\text { Upon antidepressant exposure: } \\
\text { - Children with ASD and LGD mutations had } \\
\text { increased ADOS-evaluated ASD severity }\left[F_{(1,2542)}\right. \\
=4.882 ; p=0.027] \\
\text { - Children with ASD and LGD mutations had } \\
\text { higher ADI-R verbal communication score } \\
{\left[F_{(1,2397)}=4.554 ; p=0.033\right]}\end{array}$ \\
\hline & Daud et al. (2017) & $\begin{array}{l}\text { Self-sampled buccal } \\
\text { cells (swabs) from } \\
\text { mother and neonatal }\end{array}$ & Candidate genes & PCR & $\begin{array}{l}\text { CYP1A2, CYP2C9, } \\
\text { CYP2C19, CYP2D6, } \\
\text { ABCB1, SLC6A4, } \\
\text { HTR1A, HTR1B, } \\
\text { HTR2A, and HTR3B }\end{array}$ & $\begin{array}{l}53 \text { variants in the } 10 \text { genes } \\
\text { investigated }^{\text {a }}\end{array}$ & $\begin{array}{l}\text { - No interaction found between the investigated } \\
\text { genes and antidepressant exposure on the risk of } \\
\text { congenital heart anomalies }(p>0.05)\end{array}$ \\
\hline & $\begin{array}{l}\text { Nembhard et al. } \\
\text { (2017) }\end{array}$ & $\begin{array}{l}\text { Self-sampled buccal } \\
\text { cells (swabs) from } \\
\text { parents and child }\end{array}$ & Candidate genes & Microarray & 60 genes $^{b}$ & $\begin{array}{l}872 \text { variants in the } 60 \text { genes } \\
\text { investigated }\end{array}$ & 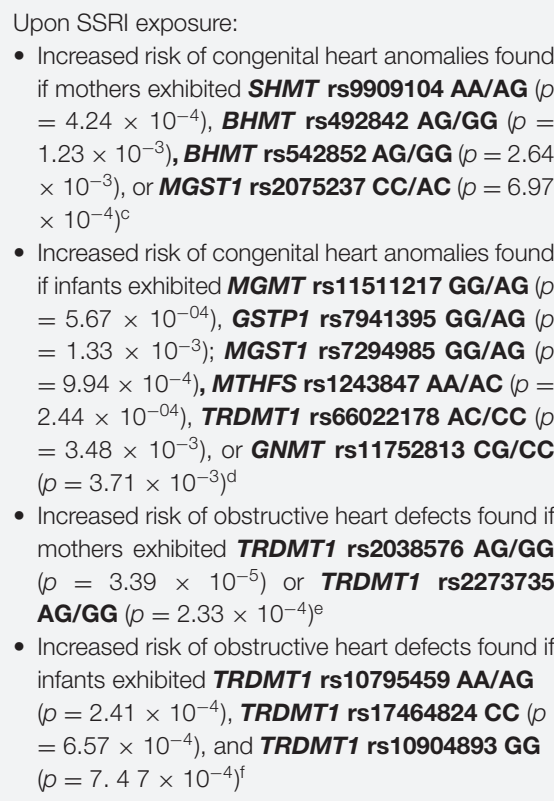 \\
\hline
\end{tabular}


TABLE 2 | Continued

\begin{tabular}{|c|c|c|c|c|c|c|}
\hline Reference & Sample tissue & Approach & Method & Gene(s) investigated & Genetic variants investigated & Pharmacogenetic outcomes \\
\hline Weikum et al. (2013) & $\begin{array}{l}\text { Neonatal whole blood } \\
\text { samples }\end{array}$ & Candidate gene & PCR & SLC6A4 & SLC6A4 long and short alleles & $\begin{array}{l}\text { - Upon SSRI exposure, children homozygous for } \\
\text { the long allele (II) of SLC6A4 had accuracy in } \\
\text { the Hearts and Flowers task inversely related to } \\
\text { the current severity of maternal depression ( } \beta \\
=-0.092 ; 95 \% \mathrm{Cl}-0.171--0.014 ; \chi^{2}=5.29 \\
p=0.021 \text {; }\end{array}$ \\
\hline $\begin{array}{l}\text { Brummelte et al. } \\
\text { (2013) }\end{array}$ & $\begin{array}{l}\text { Maternal blood and } \\
\text { cord blood samples }\end{array}$ & Candidate gene & PCR & SLC6A4 & SLC6A4 long and short alleles & $\begin{array}{l}\text { - Upon SRI exposure, mothers homozygous for } \\
\text { the long allele (II) of SLC6A4 had greater } \\
\text { expression of the } 310 \mathrm{kDa} \text { reelin band }(p=0.007)\end{array}$ \\
\hline $\begin{array}{l}\text { Oberlander et al. } \\
\text { (2010) }\end{array}$ & $\begin{array}{l}\text { Maternal blood and } \\
\text { cord blood samples }\end{array}$ & Candidate gene & PCR & SLC6A4 & SLC6A4 long and short alleles & $\begin{array}{l}\text { - No association of child or maternal SLC6A4 } \\
\text { genotypes with child behavior upon SSRI } \\
\text { exposure }\end{array}$ \\
\hline Hilli et al. (2009) & $\begin{array}{l}\text { Neonatal blood } \\
\text { samples }\end{array}$ & Candidate genes & Real-time PCR & $\begin{array}{l}\text { SLC6A4, HTR1A, } \\
\text { COMT, MAO-A, } \\
\text { CYP2C19, and } \\
\text { CYP2D6 }\end{array}$ & $\begin{array}{l}\text { SLC6A4: 5-HTTLPR and } \\
\text { 5-HTTVNTR; HTR1A: rs6295 } \\
\text { and rs6313; COMT: rs4680; } \\
\text { MAO-A: UVNTR (3, 3.5, 4, and } 5 \\
\text { copies); CYP2C19: CYP2C19*2; } \\
\text { CYP2D6: CYP2D6*3 and } \\
\text { CYP2D6*4 }\end{array}$ & 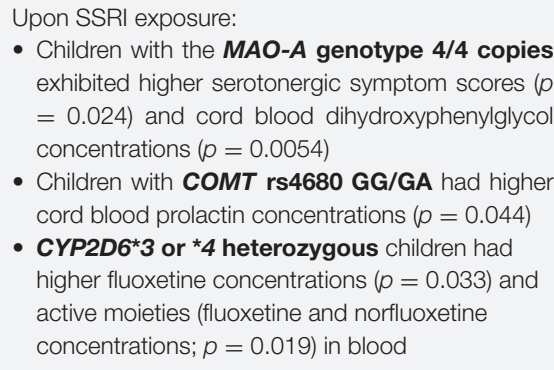 \\
\hline $\begin{array}{l}\text { Oberlander et al. } \\
\text { (2008) }\end{array}$ & $\begin{array}{l}\text { Maternal blood and } \\
\text { cord blood samples }\end{array}$ & Candidate gene & PCR & SLC6A4 & SLC6A4 long and short alleles & $\begin{array}{l}\text { Upon SSRI exposure: } \\
\text { - SLC6A4 heterozygous neonates had lower birth } \\
\text { weight }\left(F=7.45 ; p=0.008 ; \eta^{2}=0.19\right) \text { and } \\
\text { increased risk of experiencing respiratory distress } \\
(p=0.013) \\
\text { - Neonates homozygous for the long SLC6A4 } \\
\text { allele had increased risk of rapid breathing }(p \\
=0.018) \text {, respiratory distress }(p=0.043) \text {, and } \\
\text { jitteriness ( } p=0.014) \\
\text { - Neonates homozygous for the SLC6A4 short } \\
\text { allele had higher birth weight ( }(F=4.50 ; p= \\
\left.0.037 ; \eta^{2}=0.29\right) \text {, lower } 5 \text {-min Apgar }(p< \\
0.001) \text {, increased jitteriness }(p=0.026) \text {, and } \\
\text { abnormal tone }(p=0.017)\end{array}$ \\
\hline
\end{tabular}




\begin{tabular}{|c|c|c|c|c|c|c|c|}
\hline & Reference & Sample tissue & Approach & Method & Gene(s) investigated & Genetic variants investigated & Pharmacogenetic outcomes \\
\hline \multirow[t]{4}{*}{ 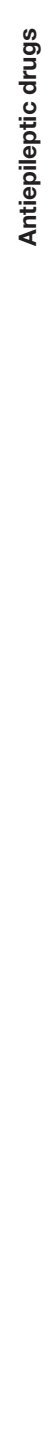 } & Jose et al. (2014) & $\begin{array}{l}\text { Peripheral blood } \\
\text { samples }\end{array}$ & Candidate genes & PCR and RFLP & $\begin{array}{l}\text { ABCB1, MTHFR, } \\
\text { CYP2C9, and } \\
\text { CYP2C19 }\end{array}$ & $\begin{array}{l}\text { ABCB1: rs3213619, rs2214102, } \\
\text { rs1202168, rs1128503, } \\
\text { rs1922242, rs2032582, and } \\
\text { rs1045642; MTHFR: rs } 1801133 \\
\text { and rs1801131; CYP2C9: } \\
\text { CYP2C9*2 and CYP2C9*3; } \\
\text { CYP2C19: CYP2C19*2 and } \\
\text { CYP2C19*3 }\end{array}$ & $\begin{array}{l}\text { Upon AED exposure: } \\
\text { - Mothers of children with congenital anomalies had } \\
\text { an increased frequency of } \boldsymbol{A B C B} \mathbf{r s} \mathbf{1 2 0 2 1 6 8} \\
\text { CC }(p=0.0032) \text { and the poor metabolizer } \\
\text { CYP2C19*2 OR }=1.639(95 \% \mathrm{Cl} 1.150-2.335 \text {; } \\
p=0.007) \text { and } \mathbf{2}^{*} \mathbf{2} \text { genotypes }(p=0.005) \\
\text { - Strong association between maternal } \boldsymbol{A B C B} \mathbf{1} \\
\text { GCT haplotype (rs2214102 G allele, } \\
\text { rs1202168C allele, rs } 1128503 \text { T allele) and } \\
\text { development of congenital anomalies }(p=2.18 \times \\
\left.10^{-5}\right)\end{array}$ \\
\hline & Azzato et al. (2010) & $\begin{array}{l}\text { Maternal blood } \\
\text { samples collected } 8 \\
\text { weeks before birth, at } \\
\text { birth, and } 6 \text { weeks after } \\
\text { birth }\end{array}$ & Candidate genes & PCR & CYP2C9 and EPHX1 & $\begin{array}{l}\text { CYP2C9: CYP2C9*2 and } \\
\text { CYP2C9*3; EPHX1: rs1051740 } \\
\text { and rs2234922 }\end{array}$ & 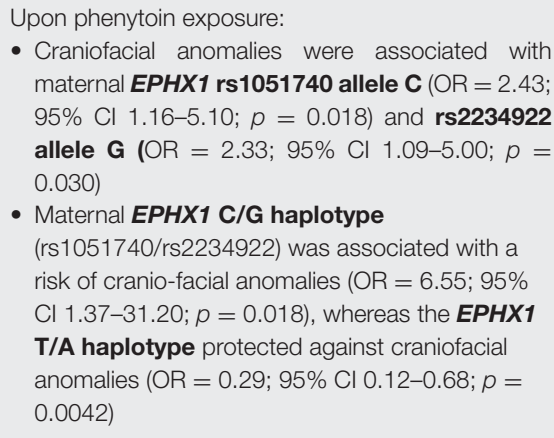 \\
\hline & Dean et al. (2007) & $\begin{array}{l}\text { Parental and neonatal } \\
\text { blood or buccal } \\
\text { samples }\end{array}$ & Candidate genes & PCR and RFLP & $\begin{array}{l}\text { MTHFR, SHMT1, MTR, } \\
\text { and MTRR }\end{array}$ & $\begin{array}{l}\text { MTHFR: rs1801133 and } \\
\text { rs1801131; SHMT: rs1979277; } \\
\text { MTR: rs1805087; MTRR: } \\
\text { rs1801394 }\end{array}$ & $\begin{array}{l}\text { Upon AED exposure: } \\
\text { - Maternal } \boldsymbol{M T H F R} \text { rs1801133 TT was associated } \\
\text { with increased risk of congenital anomalies (RR } \\
4.09 ; 95 \% \mathrm{Cl} 1.06-15.83 ; p=0.041) \text { and fetal } \\
\text { anticonvulsant syndrome (RR }=3.1 ; 95 \% \mathrm{Cl} 1.09- \\
\text { 8.81; } p=0.033 \text { ) compared to the other genotypes } \\
\text { - Children with } \boldsymbol{M T R} \text { rs1805087 GA/GG } \\
\text { genotypes had increased risk of } \\
\text { neurodevelopmental delays (OR }=1.76 ; 95 \% \mathrm{Cl} \\
1.03-3.03 ; p=0.028 \text { ) and fetal anticonvulsant } \\
\text { syndrome (OR }=1.85 ; 95 \% \mathrm{Cl} 1.13-3.02 ; p= \\
\text { 0.0089) compared to the healthy blood donor } \\
\text { controls }\end{array}$ \\
\hline & Kini et al. (2007) & $\begin{array}{l}\text { Maternal and neonatal } \\
\text { buccal samples }\end{array}$ & Candidate gene & PCR and RFLP & MTHFR & rs1801133 & $\begin{array}{l}\text { - Upon valproic acid exposure (monotherapy or } \\
\text { polytherapy), a significantly increased risk of } \\
\text { major malformations was associated with the } \\
\text { MTHFR rs1801133 CT/TT genotypes } \\
\text { compared to controls (non-exposed) CT/TT (OR } \\
=7.79 ; 95 \% \text { Cl 1.45-41.9; } p \text {-value not reported) }\end{array}$ \\
\hline
\end{tabular}


TABLE 2 | Continued

\begin{tabular}{|c|c|c|c|c|c|c|}
\hline Reference & Sample tissue & Approach & Method & Gene(s) investigated & Genetic variants investigated & Pharmacogenetic outcomes \\
\hline Dean et al. (1999) & $\begin{array}{l}\text { Parental and neonatal } \\
\text { peripheral blood } \\
\text { samples }\end{array}$ & Candidate gene & PCR and RFLP & MTHFR & rs1801133 & $\begin{array}{l}\text { - Mothers of children with fetal anticonvulsant } \\
\text { syndrome exposed to AEDs had a higher } \\
\text { frequency of } \boldsymbol{M T H F R} \text { rs } 1801133 \text { T allele }(p< \\
\text { 0.01) and TT genotype }(p<0.02) \text { compared to } \\
\text { mothers of children not exposed to AEDs }\end{array}$ \\
\hline $\begin{array}{l}\text { Van Der Voorn et al. } \\
\text { (2015) }\end{array}$ & Child blood samples & Candidate gene & Genotyping by MS & $N R 3 C 1$ and $N R 3 C 2$ & $\begin{array}{l}\text { NR3C1: rs6190 and rs6195; } \\
\text { NR3C2: rs2070951 and rs5522 }\end{array}$ & 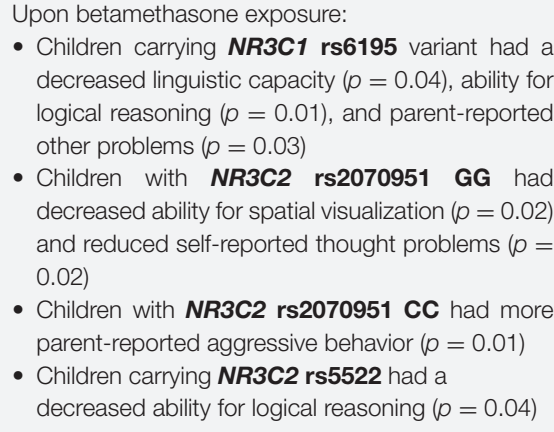 \\
\hline Haas et al. (2013) & $\begin{array}{l}\text { Maternal whole blood } \\
\text { or saliva samples and } \\
\text { neonatal cord blood or } \\
\text { saliva samples }\end{array}$ & Candidate gene & $\begin{array}{l}\text { SNP array, } \\
\text { Real-time PCR, } \\
\text { and Sanger } \\
\text { sequencing }\end{array}$ & $\begin{array}{l}\text { CYP3A4, CYP3A5, } \\
\text { CYP3A7, SULT, } \\
\text { ABCB1, NR3C1, and } \\
\text { associated pathway } \\
\text { genes }^{9}\end{array}$ & 73 SNPs $^{9}$ & 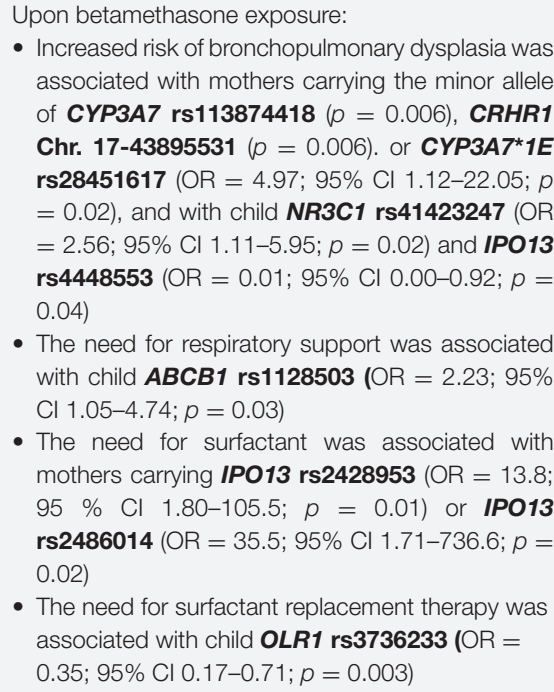 \\
\hline Oretti et al. (2009) & Cord blood samples & Candidate gene & PCR and RFLP & $\begin{array}{l}A B C B 1, N R 3 C 1 \text {, and } \\
\text { GST }\end{array}$ & $\begin{array}{l}\text { ABCB1: rs1045642, rs2032582, } \\
\text { and rs1128503; NR3C1: } \\
\text { rs41423247, rs6195, rs6189, } \\
\text { and rs6190; GSTP1: rs1695; } \\
\text { deletions in GSTM1 and GST11 }\end{array}$ & $\begin{array}{l}\text { - Upon betamethasone exposure, children with } \\
\text { respiratory distress syndrome had an increased } \\
\text { frequency of the GSTP1 rs1695 AA genotype } \\
\text { (OR = 3.758; 95\% Cl 1.16-12.17; } p=0.032)\end{array}$ \\
\hline Bertalan et al. (2008) & $\begin{array}{l}\text { Neonatal whole blood } \\
\text { or placental blood } \\
\text { samples }\end{array}$ & Candidate gene & PCR and RFLP & $N R 3 C 1$ & $\begin{array}{l}\text { rs41423247, rs6195, rs6189, } \\
\text { and rs6190 }\end{array}$ & $\begin{array}{l}\text { - No interaction found between the investigated } \\
\text { gene and dexamethasone exposure on the } \\
\text { phenotypic outcomes }(p>0.05)\end{array}$ \\
\hline
\end{tabular}

\begin{abstract}
or saliva samples and
neonatal cord blood or

saliva samples
\end{abstract}




\begin{tabular}{|c|c|c|c|c|c|c|c|}
\hline & Reference & Sample tissue & Approach & Method & Gene(s) investigated & Genetic variants investigated & Pharmacogenetic outcomes \\
\hline \multirow{7}{*}{ 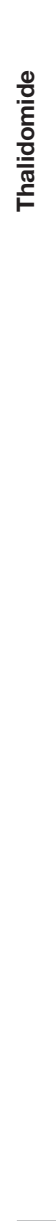 } & $\begin{array}{l}\text { Kowalski et al. } \\
\text { (2020) }\end{array}$ & $\begin{array}{l}\text { Neonatal saliva } \\
\text { samples }\end{array}$ & Candidate gene & $\begin{array}{l}\text { Targeted next } \\
\text { generation } \\
\text { sequencing }\end{array}$ & $\begin{array}{l}\text { CRBN, CUL4A, DDB1, } \\
\text { IKZF1, and IKZF3 }\end{array}$ & $\begin{array}{l}145 \text { variants found across the } 5 \\
\text { genes }\end{array}$ & $\begin{array}{l}\text { - Among individuals with thalidomide embryopathy, } \\
\text { a higher frequency of } \mathbf{C R B N} \text { rs } 1045433 \mathbf{G} \text { was } \\
\text { found in individuals with pre-axial limb anomalies } \\
(p=0.004)\end{array}$ \\
\hline & Gomes et al. (2019) & $\begin{array}{l}\text { Neonatal saliva } \\
\text { samples }\end{array}$ & Candidate gene & $\begin{array}{l}\text { Targeted next } \\
\text { generation } \\
\text { sequencing }\end{array}$ & $\begin{array}{l}\text { ESCO2, TBX5, and } \\
\text { SALL4 }\end{array}$ & $\begin{array}{l}38 \text { variants found across the } 3 \\
\text { genes }\end{array}$ & $\begin{array}{l}\text { - No interaction found between the investigated } \\
\text { genes and thalidomide exposure on the } \\
\text { susceptibility to thalidomide embryopathy ( } p> \\
0.05 \text { ) }\end{array}$ \\
\hline & Gomes et al. (2018) & $\begin{array}{l}\text { Neonatal saliva } \\
\text { samples }\end{array}$ & Candidate gene & Real-time PCR & $\begin{array}{l}\text { FGF8, FGF10, BMP4, } \\
\text { SHH, TP53, TP63, and } \\
\text { TP73 }\end{array}$ & $\begin{array}{l}\text { FGF8: rs1348870; FGF10: } \\
\text { rs900379; SHH: rs28936675; } \\
\text { BMP4: rs17563; TP53: } \\
\text { rs1042522; TP63: rs17506395; } \\
\text { TP73: rs2273953 }\end{array}$ & $\begin{array}{l}\text { No interaction found between the investigated } \\
\text { genes and thalidomide exposure on the } \\
\text { susceptibility to thalidomide embryopathy }(p> \\
\text { 0.05) }\end{array}$ \\
\hline & $\begin{array}{l}\text { Kowalski et al. } \\
\text { (2017) }\end{array}$ & $\begin{array}{l}\text { Neonatal saliva } \\
\text { samples }\end{array}$ & Candidate gene & Real-time PCR & $\begin{array}{l}\text { NOS2, PTGS2, and } \\
\text { VEGFA }\end{array}$ & $\begin{array}{l}\text { NOS2: rs2297518; PTGS2: } \\
\text { rs689465 and rs689466; } \\
\text { VEGFA: rs3025039, rs1570360, } \\
\text { rs2010963, and rs699947 }\end{array}$ & $\begin{array}{l}\text { - No interaction found between the investigated } \\
\text { genes and thalidomide exposure on the } \\
\text { susceptibility to thalidomide embryopathy }(p> \\
0.05 \text { ) }\end{array}$ \\
\hline & $\begin{array}{l}\text { Kowalski et al. } \\
\text { (2016) }\end{array}$ & $\begin{array}{l}\text { Neonatal saliva } \\
\text { samples }\end{array}$ & Candidate gene & $\begin{array}{l}\text { Real-time PCR } \\
\text { and Sanger } \\
\text { sequencing }\end{array}$ & NOS3 & $\begin{array}{l}\text { rs2070744, rs1799983, and } \\
\text { rs61722009 }\end{array}$ & $\begin{array}{l}\text { - Individuals with thalidomide embryopathy had a } \\
\text { higher frequency of } \boldsymbol{N O S} \mathbf{C} / \mathbf{4 b} \text { haplotype } \\
\text { (rs2070744/rs61722009) compared to the control } \\
\text { group; the risk of thalidomide embryopathy due } \\
\text { to the presence of this haplotype had an OR of } \\
2.57 \text { (95\% Cl: } 1.20-5.80 ; p=0.018 \text { ) }\end{array}$ \\
\hline & Vianna et al. (2016) & $\begin{array}{l}\text { Neonatal saliva } \\
\text { samples }\end{array}$ & Candidate gene & $\begin{array}{l}\text { Sanger } \\
\text { sequencing }\end{array}$ & CRBN (exons 9-11) & 8 non-exonic variants were found & $\begin{array}{l}\text { - Among individuals with thalidomide embryopathy, } \\
\text { a higher frequency of } \mathbf{C R B N} \text { rs } 1620675 \text { CC } \\
\text { (100\%) was found in individuals with neurological } \\
\text { anomalies }(n=5) \text { compared to individuals without } \\
\text { these anomalies }(p=0.004)\end{array}$ \\
\hline & Vianna et al. (2013) & $\begin{array}{l}\text { Neonatal saliva } \\
\text { samples }\end{array}$ & Candidate gene & Real-time PCR & NOS3 & rs2070744 and rs1799983 & $\begin{array}{l}\text { - Individuals with thalidomide embryopathy had a } \\
\text { higher frequency of } \mathbf{N O S 3} \text { rs2070744 C allele ( } p \\
=0.009 \text { ) and CC genotype }(p=0.031 \text { ) compared } \\
\text { to the control group } \\
\text { - The control group (with no presumed exposure } \\
\text { and no congenital anomalies) had a higher } \\
\text { frequency of } \boldsymbol{N O S 3} \text { haplotype T/G } \\
\text { (rs2070744/rs1799983; } p=0.004 \text { ) }\end{array}$ \\
\hline \multirow[t]{2}{*}{ 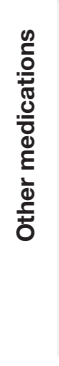 } & Bustos et al. (2017) & $\begin{array}{l}\text { Maternal blood } \\
\text { samples }\end{array}$ & Candidate gene & Real-time PCR & $\begin{array}{l}\text { CYP3A4, CYP3A5, and } \\
\text { NR3C3 }\end{array}$ & $\begin{array}{l}\text { CYP3A4: rs35599367, } \\
\text { rs2242480, and rs2740574; } \\
\text { CYP3A5: rs776746; NR3C3: } \\
\text { rs578029, rs471767, rs666553, } \\
\text { rs503362 rs500760 }\end{array}$ & $\begin{array}{l}\text { - No interaction found between the investigated } \\
\text { genes and } 7 \text {-alpha hydroxyprogesterone } \\
\text { caproate exposure on } 17 \text {-alpha } \\
\text { hydroxyprogesterone caproate plasma } \\
\text { concentrations or spontaneous preterm births ( } p \\
>0.05 \text { ) }\end{array}$ \\
\hline & $\begin{array}{l}\text { Van Der Zanden } \\
\text { et al. (2012) }\end{array}$ & Child blood samples & Candidate gene & Real-time PCR & $\begin{array}{l}\text { SRD5A2, ESR1, ESR2, } \\
\text { and ATF3 }\end{array}$ & $\begin{array}{l}\text { SRD5A2: rs523349; ESR1: } \\
\text { rs6932902; ESR2: rs2987983; } \\
\text { ATF3: rs11119982 }\end{array}$ & $\begin{array}{l}\text { - Upon estrogen exposure, the risk of hypospadias } \\
\text { was significantly associated with the presence of } \\
\text { SRD5A2 rs523349 CG }(\mathrm{RR}=2.7 ; 95 \% \mathrm{Cl} \\
1.0-7.4) \text { or GG genotypes (RR }=2.9 ; 95 \% \mathrm{Cl} \\
1.0-8.3) \text { in the affected individuals }(p<0.001)\end{array}$ \\
\hline
\end{tabular}




\section{Reference}

Perzanowski et al (2010) Sample tissue

Approach

Method

Gene(s) investigated

Genetic variants investigated

GSTP1: rs1695; GSTT2: rs2719;

Maternal and neona peripheral blood samples

and PCR
GSTT1 and GSTM1 gene deletion
NRF2, GSTP1, GST1, NRF2: rs6706649: GSTP1: and GSTM rs1695; GST11 and GSTM gene deletion

\begin{tabular}{|c|c|c|c|c|}
\hline $\begin{array}{l}\text { Shaheen et al. } \\
\text { (2010) }\end{array}$ & $\begin{array}{l}\text { Maternal whole blood } \\
\text { or saliva samples }\end{array}$ & Candidate gene & Real-time PCR & $\begin{array}{l}\text { NRF2, GSTP1, GSTT1, } \\
\text { and GSTM1 }\end{array}$ \\
\hline
\end{tabular}

The bolded values in the tables indicate the significant genes and genotypes discovered in each study a CYP1A2; rs2069521, rs2069526, rs4646425, rs4646427, rs2472304, rs2470890; CYP2C9: rs 1799853, rs1057910, rs56165452,

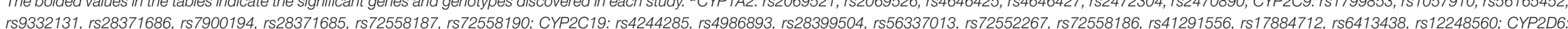
rs 16947, rs35742686, rs5030867, rs5030656, rs 1065852, rs5030863, rs5030862; ABCB1: rs 1128503, rs2032582, rs 1045642, rs2235040, rs4148739, rs1882478, rs9282564, rs 10256836; SLC6A4: rs4795541, rs57098334; HTR1A: rs 1364043, rs6295; HTR1B: rs6296, rs6298; HTR2A: rs7997012, rs6313, rs6314, rs1928040, rs6311; HTR3B: rs1176744, rs3831455; ' ${ }^{\circ}$ Genes not reported; ${ }^{c}$ Mothers: SHMT rs9909104, AG RR = 2.43 (1.46-4.03) or GG RR = 5.90 (2.13-16.24); BHMT rs492842, AG RR =2.15 (1.33-3.46) or GG RR=4.62 (1.77-11.97); BHMT rs542852, AG RR=2.06 (1.26-3.34); MGST1 rs2075237, CC RR = 7.95 (2.50-25.40) or AC RR=2.82 (1.58-5.04); ${ }^{2}$ Children: MGMT rs11511217, AG RR=2.41 (1.45-4.01); GSTP1 rs7941395, GG RR=4.71 (1.90-11.63) or AG RR=2.17 (1.38-3.41); MGST1 rs7294985, GG RR =6.10 (0.10-17.72) or AG RR=2.47 (1.45-4.21); MTHFS rs1243847, AA $R R=6.76$ (2.56-17.89) or $A C R R=2.60$ (1.60-4.23); TRDMT1 rs66022178, AC RR=1.79 (1.14-2.80) or CC RR=3.2 (1.30-7.84); GNMT rs11752813, CG RR=1.80 (1.15-2.80) or CC RR = 3.24 (1.32-7.84); ${ }^{e}$ Mothers: TRDMT rs2038576, $A G R R=4.64(2.20-9.77)$ or $G G R R=21.53$ (4.84-95.45): TRDMT rs2273735, AG RR=3.20 (1.67-6.10) or GG RR=10.24 (2.79-37.21): ${ }^{f} \mathrm{Children:}$ TRDMT1 rs 10795459, AA RR = 9.00 (2.66-30.36) or AG RR=3.0 (1.63-5.51): TRDMT1 rs17464824, CC RR=6.81 (2.07-22.56) or AC RR=2.61 (1.44-4.75); TRDMT1 rs 10904893, GG RR=6.97 (2.13-22.47) or AG RR =2.64 (1.46-4.74): ${ }^{A} A B C B 1:$ rs 1128503, rs2032582, rs 1045642; ADCY9: rs2230739; CRH: rs 12721511; CRHR1: rs1876828, rs242941; CYP3A4: rs4987159; CYP3A5*3: rs776746; CYP3A7: rs2687133; CYP3A7*2: rs2257401; GLCCI1: rs37973; IPO13: rs7412307, rs2301992, rs2301993, rs2428953, rs4448553, rs2486014, rs2240447, rs1990150, rs6671164, rs1636879; NR3C1: rs41423247; OLR1: rs3736233; STIP1/FERMT3: rs2236647: SULT1A2: rs1136703: TBX21: rs2240017: TRAPPC5/FCER2: rs28364072; CRHR1: rs4564621, rs2316763, Chr. 17- 43895531, rs242939, rs2316764, rs4277389, rs6658607; Chr. 17- 43895671, rs80249281, rs 11316767, rs 116593005, rs149144271, rs8077279, rs16940655; CYP3A7: rs45496695, rs45446698, rs 113874418, rs11568826, rs45467892, rs45575938, rs45494802, rs11568824; CYP3A7*1B: rs45465393; CYP3A7*1C: rs11568825; CYP3A7¹D: rs55798860; CYP3A7*1E: rs28451617; NR3C1: rs6189, rs6190, rs72542738, rs72481829, rs61759025, rs79138720, rs6192, rs72558023, rs72542745, rs72558022, rs13306589, rs56149945, rs1800445; CYP3A4*1B: rs2740574; CYP3A5*6: rs10264272; CYP3A5*7: rs41303343; SULT1A1*2: rs9282861; SULT1A1: rs3760091, rs750155. AED, anti-epileptic drug; ASD, autism spectrum disorder; ADI-R, Autism Diagnostic Interview-revised; ADOS, autism diagnostic observation schedule; Cl, confidence interval; LGD, likely gene-disruptive; MS, mass spectrometry; OR, odds ratio; PCR, polymerase chain reaction; RERI, relative excess risk due to interaction; RFLP, restriction fragment length polymorphism; RR, risk ratio; SNP, single nucleotide polymorphism; SRI, serotonin reuptake inhibitor; SSRI, selective serotonin reuptake inhibitor. 
(Dean et al., 1999, 2007; Kini et al., 2007). Another genetic variant investigated in the AED studies was the rs1805087 variant of MTR in children, which was associated with an increased risk of neurodevelopmental delay and fetal anticonvulsant syndrome in AED-exposed children (Dean et al., 2007). The maternal variants CYP2C19*2 and rs1202168 of $A B C B 1$, as well as the haplotype $\mathrm{G} / \mathrm{C} / \mathrm{T}$ (rs2214102/rs1202168/rs1128503) of $A B C B 1$, were also associated with an increased risk of congenital anomalies upon AED exposure (Jose et al., 2014). One study specifically examined the genetic susceptibility to phenytoin teratogenicity and found that a maternal haplotype of EPHX1 C/G (rs1051740/rs2234922) is associated with an increased risk of offspring craniofacial anomalies (Azzato et al., 2010).

\section{Glucocorticoids}

All studies on glucocorticoid exposure investigated NR3C1, the gene encoding the glucocorticoid receptor, as a susceptibility factor for glucocorticoid teratogenesis. Neonates exposed to glucocorticoids in utero and carriers of the genetic variant NR3C1 rs6195 have poorer language and cognitive abilities (Van Der Voorn et al., 2015) and an increased risk of bronchopulmonary dysplasia (Haas et al., 2013). Another gene associated with glucocorticoid adverse outcomes is $N R 3 C 2$, which encodes the mineralocorticoid receptor. Specific child genotypes (genetic variants rs2070951 and rs5522) were associated with decreased abilities in spatial visualization and logical reasoning, and increased aggressive behavior (Van Der Voorn et al., 2015). In addition, the CYP3A7 (rs113874418 and rs28451617), CRHR1 (Chr. 17-43895531), ABCB1 (rs1128503), OLR1 (rs3736233), IPO13 (rs2428953 and rs2486014), and GSTP1 (rs1695) genotypes in children were associated with respiratory complications upon glucocorticoid exposure (Haas et al., 2013).

\section{Acetaminophen}

Two studies investigated prenatal acetaminophen exposure (Perzanowski et al., 2010; Shaheen et al., 2010). The study by Perzanowski et al. (2010) found an association between the genetic variant rs1695 in GSTP1 and increased risk of wheezing at 5 years of age as well as association between the presence of at least one allele of GSTT1 and increased risk of seroatopy, in children exposed to acetaminophen during pregnancy. Both of these genes encode glutathione S-transferases (GSTs). The other study, by Shaheen et al. (2010), also found an association of the presence of at least one allele of GSTT1 in mothers with an increased risk of children presenting with wheezing or asthma at 6.5-7.5 years of age. This association was also demonstrated for the maternal variant NRF2 rs6706649 and for the presence in the mother of at least one allele of GSTM1.

\section{Sex Hormones}

In the one study investigating prenatal estrogen exposure, the genetic variant rs523349 located in SRD5A2 was associated with an increased risk of hypospadias (Van Der Zanden et al., 2012). Bustos et al. (2017) investigated the potential effect of CYP3A4, CYP3A5, and NR3C3 on the plasma concentrations of 7-alpha hydroxyprogesterone caproate (a progestin) and the interaction of these genes with the drug in spontaneous premature delivery. However, the authors did not find any gene-outcome associations (Bustos et al., 2017).

\section{DISCUSSION}

\section{Key Findings and Methodological Limitations}

Teratogenesis is a complex event in which different factors affect the outcome in an individual. Knowledge of the teratogenic potential of many drugs is still limited and disputed, and no unique list of likely teratogenic drugs exists. In contrast to Wilson's other principles of teratogenesis (Wilson, 1977), the evaluation of genetic susceptibility to teratogens represents a challenge in regards to both research methodologies and strategies, and the interpretation and application of results. Therefore, we performed a systematic review to summarize and compare the findings of studies investigating the association of the mother and/or child's genetic background with the risk of congenital anomalies or developmental abnormalities due to prenatal exposure to drugs with teratogenic potential.

In studies investigating SRI exposure, SLC6A4, which encodes the serotonin transporter, was the gene most commonly associated with adverse fetal outcomes. Serotonin has several important roles during embryonic and fetal brain development, including neuronal maturation, migration, synaptogenesis, and differentiation of neural crest cells associated with facial and cardiac development (Brummelte et al., 2017). Brummelte et al. (2017) suggest that these important roles of serotonin may explain why SSRIs and variations in SLC6A4 could impair embryonic and fetal development.

Thalidomide is one of the oldest known teratogenic agents, causing thalidomide embryopathy (Vargesson, 2009). The reviewed studies on thalidomide focused on examining genes encoding proteins important in angiogenesis and oxidative stress, or proteins that are part of the CLR4-Cereblon complex, all of which are known to be associated with thalidomide teratogenicity (Therapontos et al., 2009; Ito et al., 2010; Vianna et al., 2013, 2016; Ha et al., 2016; Kowalski et al., 2016, 2017, 2020).

The reviewed studies on AEDs mainly investigated MTHFR, which is associated with folate metabolism (Dean et al., 1999, 2007; Kini et al., 2007; Jose et al., 2014). Folate is essential for both methylation and DNA synthesis, and folate metabolism is important for normal fetal development (Nazki et al., 2014). It has been demonstrated that AED treatment is associated with lower mean serum folate levels, but the mechanisms underlying this association remain speculative and might include interferences with diet, absorption, plasma binding, cellular metabolism, and renal excretion (Linnebank et al., 2011). Therefore, examining functional polymorphisms in genes of proteins involved in the folate pathway could be highly relevant to better understanding the differential susceptibility of individuals to the teratogenic effects of AEDs.

Glucocorticoids are usually related to the stimulation of fetal growth and organ maturation. However, an excess of synthetic glucocorticoids can negatively affect neonatal development (Clifton et al., 2017). All of the reviewed studies 
on glucocorticoid exposure investigated NR3C1, which encodes the glucocorticoid receptor, as a susceptibility factor for glucocorticoid teratogenesis. As glucocorticoids act via this receptor, the presence of genetic variants that change the glucocorticoid binding site could affect the biological functioning of the receptor in fetal development (Adcock and Mumby, 2016; Busada and Cidlowski, 2017).

The two studies investigating the genetic susceptibility to acetaminophen teratogenesis found that genes encoding glutathione S-transferases (GSTs) are associated with the presence of child wheezing and asthma (Perzanowski et al., 2010; Shaheen et al., 2010). GSTs are involved in acetaminophen pharmacokinetics and are important for the prevention of lung damage through the detoxification of reactive oxygen species generated by inflammation, infection, and other exposures (Zhao and Pickering, 2011). Therefore, the effects of acetaminophen exposure on respiratory outcomes have been suggested to be modified by GST activity (Dai et al., 2020).

The use of estrogens and other hormones during pregnancy has been associated with male reproductive disorders, such as hypospadias and cryptorchidism (Storgaard et al., 2006). This observation was replicated by Van Der Zanden et al. (2012), who found that SRD5A2 (variant rs523349) is associated with hypospadias upon prenatal estrogen exposure.

Although a large number of drugs were included in our list of search terms (Appendices 1, 2), only a few of these drugs were investigated in prenatal pharmacogenetic studies, even among the drugs with known teratogenic properties. In particular, of the twelve known teratogenic drugs in Appendix 1, nine were not investigated in either of the reviewed studies (including ACE inhibitors, antineoplastic agents, diethylstilbestrol, fingolimod, lithium, misoprostol, mycophenolate, retinoids, and warfarin). Another limitation of most of the studies in this review was a small sample size. In addition, some studies re-used the samples across multiple projects, which can decrease the power for finding strong and reliable associations. Small sample sizes also limit the power to study medications at a substance level. This is unfortunate as even small variations in chemical structure may impact the embryo-fetal toxicity of the drug. For instance, four out of the five reviewed studies on AEDs, were performed on medications with important structural, pharmacological and teratogenic differences, such as lamotrigine (Mufson, 2018) and valproic acid (Rosenberg, 2007). Group-level examinations may be acceptable if the medications within the specific group are expected to exhibit similar mechanisms of action and similar embryo-fetal toxicity (e.g., SSRIs). Furthermore, most studies used a candidate gene approach, whereas only one study applied a genome-wide approach (Ackerman et al., 2017). Candidate gene approaches limit the comparison of results and the application of the results in future studies due to the a priori selection of a few genes. This may be an appropriate approach if there is a strong hypothesis of certain genes being involved but, in most cases, current knowledge is too sparse to support this as the preferred approach. Therefore, comprehensive genomewide association studies (GWASs) may considerably broaden the search for potentially interesting genes.

\section{Future Directions: Toward Personalized Medicine}

To realize the potential of studies within genetic teratology, it is important to work toward overcoming current limitations and to improve our approaches within this field. Such efforts may ultimately enable clinical implementation of prenatal pharmacogenetic screening. This will enhance the clinical relevance and drive the field toward personalized medicine for pregnant women in need of drugs.

Teratogenesis is a complex, multifactorial process and an understanding of both genetic and environmental factors is pivotal to predicting the effects of a teratogen (Kowalski et al., 2020). Furthermore, multiple genetic variants with small individual effects are likely to be associated with teratogenic susceptibility (Edwards et al., 2013). Upon exposure to a teratogen, the synergistic effect of the genetic and pharmaceutical factors may cause teratogenesis. It is essential to perform genome-wide investigations to account for the multiple genetic variants associated with a phenotypic outcome of prenatal drug exposure. If only a candidate gene approach is feasible, the study should have a strong hypothesis for selecting the gene(s). Such a rationale may be founded on biological plausibility derived from animal and in vitro studies, or known involvement of the genes in drug metabolism.

Investigation of complex teratogenic processes, likely involving non-linear and non-additive interactions of multiple genetic and environmental factors, are further supported by the development of novel methods within bioinformatics (Weber et al., 2019). Analyzing and deciphering such higherorder interactions prompts the application of machine learning models, which can detect patterns and predict drug teratogenicity and child outcomes. For example, machine learning has been applied in the investigation of non-teratogenic birth defect profiles, predicting groups at high risk of congenital heart defects (Luo et al., 2017), drugs of great concern during pregnancy (Ailes et al., 2020), and the probability of a drug being teratogenic (Challa et al., 2020).

Health authorities and funding bodies play a pivotal role in facilitating further advances in research on genetic teratology. Such institutions should take on the responsibility of supporting research, particularly for drugs that have had conflicting results, such as antidepressants and AEDs, but also ADHD, antithyroid, and HIV drugs. Support can be provided by making more research funding available, such as through initiatives like the IMI ConcePTION project (ConcePTION, 2020). In addition, health authorities should consider requesting pharmacogenetic studies from the market authorization holders if gene polymorphisms are expected to play a significant role in drug teratogenicity.

Finally, genetic profiling of human fetuses is essential to translate findings from studies on genetic teratology into clinical diagnostics. However, access to tissue specimens during pregnancy is often limited due to the risk of affecting normal development or causing miscarriages by invasive sampling techniques (Salomon et al., 2019). Therefore, it is important 
that novel methods, such as non-invasive prenatal testing (NIPT; Lau et al., 2012; Allyse et al., 2015), are developed and applied clinically. Moreover, developing and implementing personalized medicine approaches like those described above in clinical practice, will require multidisciplinary teams with clinical geneticists, bioinformaticians, obstetricians, and reproductive toxicologists.

\section{CONCLUSION}

In summary, this systematic review identified 29 studies on human genetic variation associated with the teratogenesis of several drugs. Antidepressants, AEDs, glucocorticoids, and thalidomide were the most investigated drugs. The most convincing findings were genetic variants in SLC6A4, MTHFR, and NR3C1 being associated with drug teratogenicity from antidepressants, AEDs, and glucocorticoids, respectively. Future studies on genetic teratology should aim to consider the biological pathways and molecular mechanisms of teratogens more broadly, and take into consideration the complexity of the multifactorial teratogenic process. This approach requires increased sample sizes, the application of genome-wide approaches, and international funding initiatives. To translate the research into the clinic, application of novel methodologies for the collection and examination of fetal DNA is encouraged, as well as the use of machine learning methods and implementation of multidisciplinary teams. Taken together, these efforts may ultimately promote early interventions and possibly minimize the teratogenic impact of maternal drug use during pregnancy on the fetus.

\section{REFERENCES}

Ackerman, S., Schoenbrun, S., Hudac, C., and Bernier, R. (2017). Interactive effects of prenatal antidepressant exposure and likely gene disrupting mutations on the severity of autism spectrum disorder. J. Autism Dev. Disord. 47, 3489-3496. doi: 10.1007/s10803-017-3246-6

Adcock, I. M., and Mumby, S. (2016). "Glucocorticoids," in Handbook of Experimental Pharmacology, eds. C. Page, and P. Barnes (Cham: Springer New York LLC), 171-196.

Ailes, E. C., Zimmerman, J., Lind, J. N., Fan, F., Shi, K., Reefhuis, J., et al. (2020). Using supervised learning methods to develop a list of prescription medications of greatest concern during pregnancy. Matern. Child Health J. 24, 901-910. doi: 10.1007/s10995-020-02942-2

Allyse, M., Minear, M. A., Berson, E., Sridhar, S., Rote, M., Hung, A., et al. (2015). Non-invasive prenatal testing: a review of international implementation and challenges. Int. J. Womens. Health 7, 113-126. doi: 10.2147/IJWH.S67124

Azzato, E. M., Chen, R. A., Wacholder, S., Chanock, S. J., Klebanoff, M. A., and Caporaso, N. E. (2010). Maternal EPHX1 polymorphisms and risk of phenytoin-induced congenital malformations. Pharmacogenet. Genomics 20, 58-63. doi: 10.1097/FPC.0b013e328334b6a3

Bakker, M. K., Kerstjens-Frederikse, W. S., Buys, C. H. C. M., De Walle, H. E. K., and De Jong-van Den Berg, L. T. W. (2010). First-trimester use of paroxetine and congenital heart defects: a population-based case-control study. Birth Defects Res. A Clin. Mol. Teratol. 88, 94-100. doi: 10.1002/bdra.20641

Bertalan, R., Patocs, A., Vasarhelyi, B., Treszl, A., Varga, I., Szabo, E., et al. (2008). Association between birth weight in preterm neonates and the BclI polymorphism of the glucocorticoid receptor gene. J. Steroid Biochem. Mol. Biol. 111, 91-94. doi: 10.1016/j.jsbmb.2008.05.002

\section{DATA AVAILABILITY STATEMENT}

The original contributions presented in the study are included in the article/Supplementary Material, further inquiries can be directed to the corresponding author/s.

\section{AUTHOR CONTRIBUTIONS}

JAG, EWO, LS-F, and HMEN contributed to the study conception and design. JAG and EWO performed the literature searches and data analyses. JAG wrote the manuscript. All authors critically revised the manuscript.

\section{FUNDING}

This work has been conducted as part of the PharmaTox QLS INTPART project, which enabled Julia do Amaral Gomes to have a research visit at the Pharmacoepidemiology and Drug Safety Research Group, Department of Pharmacy, Faculty of Mathematics and Natural Sciences, University of Oslo, Oslo, Norway. Prof. Hedvig Nordeng is supported by a European Research Council Starting Grant Drugs In Pregnancy (Grant Number 639377). FV is recipient of a CNPq scholarship grant (Grant Number CNPq 312993 / 2017-0).

\section{SUPPLEMENTARY MATERIAL}

The Supplementary Material for this article can be found online at: https://www.frontiersin.org/articles/10.3389/fgene. 2021.645555/full\#supplementary-material

Bleichrodt, N., and van den Berg, R. H. (2000). Multicultural Capacity Test: Intermediate Level (MCT-M) Manual. Amsterdam: NOA-VU.

Bromley, R. L., Weston, J., and Marson, A. G. (2017). Maternal use of antiepileptic agents during pregnancy and major congenital malformations in children. $J$. Am. Med. Assoc. 318, 1700-1701. doi: 10.1001/jama.2017.14485

Brummelte, S., Galea, L. A. M., Devlin, A. M., and Oberlander, T. F. (2013). Antidepressant use during pregnancy and serotonin transporter genotype (SLC6A4) Affect newborn serum reelin levels. Dev. Psychobiol. 55, 518-529. doi: 10.1002/dev.21056

Brummelte, S., Mc Glanaghy, E., Bonnin, A., and Oberlander, T. F. (2017). Developmental changes in serotonin signaling: Implications for early brain function, behavior and adaptation. Neuroscience 342, 212-231. doi: 10.1016/j.neuroscience.2016.02.037

Busada, J. T., and Cidlowski, J. A. (2017). "Mechanisms of Glucocorticoid Action During Development," in Current Topics in Developmental Biology, eds D. Forrest and S. Tsai (San Diego, CA: Academic Press Inc.), 147-170.

Bustos, M. L., Caritis, S. N., Jablonski, K. A., Reddy, U. M., Sorokin, Y., Manuck, T., et al. (2017). The association among cytochrome P450 3A, progesterone receptor polymorphisms, plasma 17-alpha hydroxyprogesterone caproate concentrations, and spontaneous preterm birth. Am. J. Obstet. Gynecol. 217, 369.e1-369.e9. doi: 10.1016/j.ajog.2017.05.019

Cassina, M., Salviati, L., Di Gianantonio, E., and Clementi, M. (2012). Genetic susceptibility to teratogens: state of the art. Reprod. Toxicol. 34, 186-191. doi: 10.1016/j.reprotox.2012.05.004

Challa, A. P., Beam, A. L., Shen, M., Peryea, T., Lavieri, R. R., Lippmann, E. S., et al. (2020). Machine learning on drug-specific data to predict small molecule teratogenicity. Reprod. Toxicol. 95, 148-158. doi: 10.1016/j.reprotox.2020.05.004 
Clifton, V. L., Cuffe, J., Moritz, K. M., Cole, T. J., Fuller, P. J., Lu, N. Z., et al. (2017). Review: the role of multiple placental glucocorticoid receptor isoforms in adapting to the maternal environment and regulating fetal growth. Placenta 54, 24-29. doi: 10.1016/j.placenta.2016.12.017

ConcePTION. (2020). IMI ConcePTION Project. Available online at: https://www. imi-conception.eu/ (accessed December 10, 2020).

Corti, S., Pileri, P., Mazzocco, M. I., Mand,ò, C., Moscatiello, A. F., Cattaneo, D., et al. (2019). Neonatal outcomes in maternal depression in relation to intrauterine drug exposure. Front. Pediatr. 7:309. doi: 10.3389/fped.2019.00309

Dai, X., Dharmage, S. C., Abramson, M. J., Erbas, B., Bennett, C. M., Svanes, C., et al. (2020). Early life acetaminophen exposure, glutathione S-transferase genes, and development of adolescent asthma in a high-risk birth cohort. J. Allergy Clin. Immunol. 146, 1035-1044.e12. doi: 10.1016/j.jaci.2020.03.027

Daud, A. N. A., Bergman, J. E. H., Kerstjens-Frederikse, W. S., Van Der Vlies, P., Hak, E., Berger, R. M. F., et al. (2017). Prenatal exposure to serotonin reuptake inhibitors and congenital heart anomalies: an exploratory pharmacogenetics study. Pharmacogenomics 18, 987-1001. doi: 10.2217/pgs-2017-0036

Dean, J., Robertson, Z., Reid, V., Wang, Q. D., Hailey, H., Moore, S., et al. (2007). Fetal anticonvulsant syndromes and polymorphisms in MTHFR, MTR, and MTRR. Am. J. Med. Genet. A 143, 2303-2311. doi: 10.1002/ajmg.a.31914

Dean, J. C. S., Moore, S. J., Osborne, A., Howe, J., and Turnpenny, P. D. (1999). Fetal anticonvulsant syndrome and mutation in the maternal MTHFR gene. Clin. Genet. 56, 216-220. doi: 10.1034/j.1399-0004.1999.560306.x

Edwards, S. L., Beesley, J., French, J. D., and Dunning, M. (2013). Beyond GWASs: illuminating the dark road from association to function. Am. J. Hum. Genet. 93, 779-797. doi: 10.1016/j.ajhg.2013.10.012

Engeland, A., Bramness, J. G., Daltveit, A. K., Rønning, M., Skurtveit, S., and Furu, K. (2008). Prescription drug use among fathers and mothers before and during pregnancy. A population-based cohort study of 106000 pregnancies in Norway 2004-2006. Br. J. Clin. Pharmacol. 65, 653-660. doi: $10.1111 / \mathrm{j} .1365-2125.2008 .03102 . x$

Eriksen, M. B., and Frandsen, T. F. (2018). The impact of patient, intervention, comparison, outcome (Pico) as a search strategy tool on literature search quality: a systematic review. J. Med. Libr. Assoc. 106, 420-431. doi: 10.5195/JMLA.2018.345

Gomes, J., do, A., Kowalski, T. W., Fraga, L. R., Tovo-Rodrigues, L., Sanseverino, M. T. V., et al. (2018). Genetic susceptibility to thalidomide embryopathy in humans: Study of candidate development genes. Birth Defects Res. 110, 456-461. doi: 10.1002/bdr2.1163

Gomes, JdA., Kowalski, T. W., Fraga, L. R., Macedo, G. S., Sanseverino, M. T. V., Schuler-Faccini, L., et al. (2019). The role of ESCO2, SALL4 and TBX5 genes in the susceptibility to thalidomide teratogenesis. Sci. Rep. 9:11413. doi: 10.1038/s41598-019-47739-8

Griffiths, J. A., Scialdone, A., and Marioni, J. C. (2018). Using single-cell genomics to understand developmental processes and cell fate decisions. Mol. Syst. Biol. 14:e8046. doi: 10.15252/msb.20178046

Ha, J. M., Jin, S. Y., Lee, H. S., Shin, H. K., Lee, D. H., Song, S. H., et al. (2016). Regulation of retinal angiogenesis by endothelial nitric oxide synthase signaling pathway. Korean J. Physiol. Pharmacol. 20, 533-538. doi: 10.4196/kjpp.2016.20.5.533

Haas, D. M., Dantzer, J., Lehmann, A. S., Philips, S., Skaar, T. C., McCormick, C. L., et al. (2013). The impact of glucocorticoid polymorphisms on markers of neonatal respiratory disease after antenatal betamethasone administration. Am. J. Obstet. Gynecol. 208, 215.e1-215.e6. doi: 10.1016/j.ajog.2012.12.031

Harris, B. S., Bishop, K. C., Kemeny, H. R., Walker, J. S., Rhee, E., and Kuller, J. A. (2017). Risk factors for birth defects. Obstet. Gynecol. Surv. 72, 123-135. doi: 10.1097/OGX.0000000000000405

Hilli, J., Heikkinen, T., Rontu, R., Lehtimäki, T., Kishida, I., Aklillu, E., et al. (2009). MAO-A and COMT genotypes as possible regulators of perinatal serotonergic symptoms after in utero exposure to SSRIs. Eur. Neuropsychopharmacol. 19, 363-370. doi: 10.1016/j.euroneuro.2009.01.006

Iossifov, I., O’Roak, B. J., Sanders, S. J., Ronemus, M., Krumm, N., Levy, D., et al. (2014). The contribution of de novo coding mutations to autism spectrum disorder. Nature 515, 216-221. doi: 10.1038/nature13908

Ito, T., Ando, H., Suzuki, T., Ogura, T., Hotta, K., Imamura, Y., et al. (2010). Identification of a primary target of thalidomide teratogenicity. Science. 327, 1345-1350. doi: 10.1126/science.1177319
Jose, M., Banerjee, M., Mathew, A., Bharadwaj, T., Vijayan, N., and Thomas, S. (2014). Pharmacogenetic evaluation of ABCB1, Cyp2C9, Cyp2C19 and methylene tetrahydrofolate reductase polymorphisms in teratogenicity of antiepileptic drugs in women with epilepsy. Ann. Indian Acad. Neurol. 17, 259-266. doi: 10.4103/0972-2327.138475

Khare, R., Leaman, R., and Lu, Z. (2014). Accessing biomedical literature in the current information landscape. Methods Mol Biol. 1159, 11-31. doi: 10.1007/978-1-4939-0709-0_2

Kini, U., Lee, R., Jones, A., Smith, S., Ramsden, S., Fryer, A., et al. (2007). Influence of the MTHFR genotype on the rate of malformations following exposure to antiepileptic drugs in utero. Eur. J. Med. Genet. 50, 411-420. doi: 10.1016/j.ejmg.2007.08.002

Kowalski, T. W., Fraga, L. R., Tovo-Rodrigues, L., Sanseverino, M. T. V., Hutz, M. H., Schuler-Faccini, L., et al. (2016). New Findings in eNOS gene and Thalidomide Embryopathy Suggest pre-transcriptional effect variants as susceptibility factors. Sci. Rep. 6:23404. doi: 10.1038/srep23404

Kowalski, T. W., Fraga, L. R., Tovo-Rodrigues, L., Sanseverino, M. T. V., Hutz, M. H., Schuler-Faccini, L., et al. (2017). Angiogenesis-related genes and thalidomide teratogenesis in humans: an approach on genetic variation and review of past in vitro studies. Reprod. Toxicol. 70, 133-140. doi: 10.1016/j.reprotox.2017.01.012

Kowalski, T. W., Gomes, JdA., Garcia, G. B. C., Fraga, L. R., Paixao-Cortes, V. R., Recamonde-Mendoza, M., et al. (2020). CRL4-Cereblon complex in Thalidomide Embryopathy: a translational investigation. Sci. Rep. 10:851. doi: 10.1038/s41598-020-57512-x

Laine, K., Heikkinen, T., Ekblad, U., and Kero, P. (2003). Effects of exposure to selective serotonin reuptake inhibitors during pregnancy on serotonergic symptoms in newborns and cord blood monoamine and prolactin concentrations. Arch. Gen. Psychiatry 60, 720-726. doi: 10.1001/archpsyc.60.7.720

Lau, T. K., Chan, M. K., Salome Lo, P. S., Connie Chan, H. Y., Kim Chan, W. S., Koo, T. Y., et al. (2012). Clinical utility of noninvasive fetal trisomy (NIFTY) test early experience. J. Matern. Neonatal Med. 25, 1856-1859. doi: $10.3109 / 14767058.2012 .678442$

Liberati, A., Altman, D. G., Tetzlaff, J., Mulrow, C., Gøtzsche, P. C., Ioannidis, J. P., et al. (2009). The PRISMA statement for reporting systematic reviews and meta-analyses of studies that evaluate health care interventions: explanation and elaboration. PLoS Med. 6:e1000100. doi: 10.1371/journal.pmed.1000100

Linnebank, M., Moskau, S., Semmler, A., Widman, G., Stoffel-Wagner, B., Weller, M., et al. (2011). Antiepileptic drugs interact with folate and vitamin B12 serum levels. Ann. Neurol. 69, 352-359. doi: 10.1002/ana.22229

Luo, Y., Li, Z., Guo, H., Cao, H., Song, C., Guo, X., et al. (2017). Predicting congenital heart defects: a comparison of three data mining methods. PLoS One 12:e177811. doi: 10.1371/journal.pone.0177811

Momen, N. C., Ernst, A., Arendt, L. H., Olsen, J., Li, J., Gissler, M., et al. (2017). Maternal cancer and congenital anomalies in children \pm a Danish nationwide cohort study. PLoS One 12:e173355. doi: 10.1371/journal.pone.0173355

Mufson, J. M. (2018). Lamotrigine: pharmacology, clinical utility, and new safety concerns. Am. J. Psychiatry 13, 2-4. doi: 10.1176/appi.ajp-rj.2018. 131201

Nazki, F. H., Sameer, A. S., and Ganaie, B. A. (2014). Folate: Metabolism, genes, polymorphisms and the associated diseases. Gene 533, 11-20. doi: 10.1016/j.gene.2013.09.063

Nembhard, W. N., Tang, X., Hu, Z., Macleod, S., Stowe, Z., and Webber, D. (2017). Maternal and infant genetic variants, maternal periconceptional use of selective serotonin reuptake inhibitors, and risk of congenital heart defects in offspring: population based study. BMJ 356. doi: 10.1136/bmj.j832

Oberlander, T. F., Bonaguro, R. J., Misri, S., Papsdorf, M., Ross, C. J. D., and Simpson, E. M. (2008). Infant serotonin transporter (SLC6A4) promoter genotype is associated with adverse neonatal outcomes after prenatal exposure to serotonin reuptake inhibitor medications. Mol. Psychiatry 13, 65-73. doi: 10.1038/sj.mp.4002007

Oberlander, T. F., Papsdorf, M., Brain, U. M., Misri, S., Ross, C., and Grunau, R. E. (2010). Prenatal effects of selective serotonin reuptake inhibitor antidepressants, serotonin transporter promoter genotype (SLC6A4), and maternal mood on child behavior at 3 years of age. Arch. Pediatr. Adolesc. Med. 164, 444-451. doi: 10.1001/archpediatrics.2010.51 
Olesen, C., Steffensen, F. H., Nielsen, G. L., De Jong-Van Den Berg, L., Olsen, J., Sørensen, H. T., et al. (1999). Drug use in first pregnancy and lactation: a population-based survey among Danish women. Eur. J. Clin. Pharmacol. 55, 139-144. doi: 10.1007/s002280050608

Oretti, C., Marino, S., Mosca, F., Colnaghi, M. R., De Iudicibus, S., Drigo, I., et al. (2009). Glutathione-S-transferase-P1 I105V polymorphism and response to antenatal betamethasone in the prevention of respiratory distress syndrome. Eur. J. Clin. Pharmacol. 65, 483-491. doi: 10.1007/s00228-009-0617-8

Ostensen, M. (1992). Treatment with immunosuppressive and disease modifying drugs during pregnancy and lactation. Am. J. Reprod. Immunol.28, 148-152. doi: 10.1111/j.1600-0897.1992.tb00778.x

Ouzzani, M., Hammady, H., Fedorowicz, Z., and Elmagarmid, A. (2016). Rayyan-a web and mobile app for systematic reviews. Syst. Rev. 5:210. doi: 10.1186/s13643-016-0384-4

Perzanowski, M. S., Miller, R. L., Tang, D., Ali, D., Garfinkel, R. S., Chew, G. L., et al. (2010). Prenatal acetaminophen exposure and risk of wheeze at age 5 years in an urban low-income cohort. Thorax 65, 118-123. doi: 10.1136/thx.2009.121459

Piña, B., Navarro, L., Barata, C., Raldúa, D., Martínez, R., and Casado, M. (2018). "Omics in zebrafish teratogenesis," in Methods in Molecular Biology, eds. L. Felix (New York, NY: Humana Press Inc.), 421-441.

Richardson, W. S., Wilson, M. C., Nishikawa, J., and Hayward, R. S. (1995). The well-built clinical question: a key to evidence-based decisions. ACP J. Club 123, A12-13.

Rosenberg, G. (2007). The mechanisms of action of valproate in neuropsychiatric disorders: can we see the forest for the trees? Cell. Mol. Life Sci. 64, 2090-2103. doi: 10.1007/s00018-007-7079-x

Salomon, L. J., Sotiriadis, A., Wulff, C. B., Odibo, A., and Akolekar, R. (2019). Risk of miscarriage following amniocentesis or chorionic villus sampling: systematic review of literature and updated meta-analysis. Ultrasound Obstet. Gynecol. 54, 442-451. doi: 10.1002/uog.20353

Schaefer, C., Peters, P. W. J., and Miller, R. K. (2014). Drugs During Pregnancy and Lactation, 3rd edn. London: Academic Press.

Shaheen, S. O., Newson, R. B., Ring, S. M., Rose-Zerilli, M. J., Holloway, J. W., and Henderson, A. J. (2010). Prenatal and infant acetaminophen exposure, antioxidant gene polymorphisms, and childhood asthma. J. Allergy Clin. Immunol. 126, 1141-8.e7. doi: 10.1016/j.jaci.2010.08.047

Sitkin, N. A., Ozgediz, D., Donkor, P., and Farmer, D. L. (2015). Congenital anomalies in low- and middle-income countries: the unborn child of global surgery. World J. Surg. 39, 36-40. doi: 10.1007/s00268-014-2714-9

Song, R., and Yosypiv, I. V. (2011). Genetics of congenital anomalies of the kidney and urinary tract. Pediatr. Nephrol. 26, 353-364. doi: 10.1007/s00467-010-1629-4

Sternbach, H. (1991). The serotonin syndrome. Am. J. Psychiatry 148, 705-713. doi: 10.1176/ajp.148.6.705

Storgaard, L., Bonde, J. P., and Olsen, J. (2006). Male reproductive disorders in humans and prenatal indicators of estrogen exposure: a review of published epidemiological studies. Reprod. Toxicol. 21, 4-15. doi: 10.1016/j.reprotox.2005.05.006

Therapontos, C., Erskine, L., Gardner, E. R., Figg, W. D., and Vargesson, N. (2009). Thalidomide induces limb defects by preventing angiogenic outgrowth during early limb formation. Proc. Natl. Acad. Sci. U. S. A. 106, 8573-8578. doi: 10.1073/pnas.0901505106

Van Der Voorn, B., Wit, J. M., Van Der Pal, S. M., Rotteveel, J., and Finken, M. J. J. (2015). Antenatal glucocorticoid treatment and polymorphisms of the glucocorticoid and mineralocorticoid receptors are associated with IQ and behavior in young adults born very preterm. J. Clin. Endocrinol. Metab. 100, 500-507. doi: 10.1210/jc.2014-2843

Van Der Zanden, L. F. M., Galesloot, T. E., Feitz, W. F. J., Brouwers, M. M., Shi, M., Knoers, N. V. A. M., et al. (2012). Exploration of gene-environment interactions, maternal effects and parent of origin effects in the etiology of hypospadias. J. Urol. 188, 2354-2360. doi: 10.1016/j.juro.2012.08.033

Vargesson, N. (2009). Thalidomide-induced limb defects: Resolving a 50-year-old puzzle. BioEssays 31, 1327-1336. doi: 10.1002/bies.200900103

Vianna, F. S. L., Fraga, L. R., Tovo-Rodrigues, L., Tagliani-Ribeiro, A., Biondi, F., Maximino, C. M., et al. (2013). Polymorphisms in the endothelial nitric oxide synthase gene in thalidomide embryopathy. Nitric Oxide Biol. Chem. 35, 89-92. doi: 10.1016/j.niox.2013.09.002

Vianna, F. S. L., Kowalski, T. W., Tovo-Rodrigues, L., Tagliani-Ribeiro, A., Godoy, B. A., Fraga, L. R., et al. (2016). Genomic and in silico analyses of CRBN gene and thalidomide embryopathy in humans. Reprod. Toxicol. 66, 99-106. doi: 10.1016/j.reprotox.2016.10.003

Weber, K. A., Yang, W., Carmichael, S. L., Padula, A. M., and Shaw, G. M. (2019). A machine learning approach to investigate potential risk factors for gastroschisis in California. Birth Defects Res. 111, 212-221. doi: 10.1002/bdr2.1441

Weikum, W. M., Brain, U., Chau, C. M. Y., Grunau, R. E., Boyce, W. T., Diamond, A., et al. (2013). Prenatal serotonin reuptake inhibitor (SRI) antidepressant exposure and serotonin transporter promoter genotype (SLC6A4) influence executive functions at 6 years of age. Front. Cell. Neurosci. 7:180. doi: 10.3389/fncel.2013.00180

Williams, K., Carson, J., and Lo, C. (2019). Genetics of congenital heart disease. Biomolecules 9:879. doi: 10.3390/biom9120879

Wilson, J. (1977). Handbook of Teratology, Volume 1: General Principles and Etiology. New York, NY; London: Plenum Press.

Yalcin, D., Hakguder, Z. M., and Otu, H. H. (2015). Bioinformatics approaches to single-cell analysis in developmental biology. Mol. Hum. Reprod. 22, 182-192. doi: 10.1093/molehr/gav050

Zhao, L., and Pickering, G. (2011). Paracetamol metabolism and related genetic differences. Drug Metab. Rev. 43, 41-52. doi: 10.3109/03602532.2010.527984

Conflict of Interest: The authors declare that the research was conducted in the absence of any commercial or financial relationships that could be construed as a potential conflict of interest.

Copyright (C) 2021 Gomes, Olstad, Kowalski, Gervin, Vianna, Schüler-Faccini and Nordeng. This is an open-access article distributed under the terms of the Creative Commons Attribution License (CC BY). The use, distribution or reproduction in other forums is permitted, provided the original author(s) and the copyright owner(s) are credited and that the original publication in this journal is cited, in accordance with accepted academic practice. No use, distribution or reproduction is permitted which does not comply with these terms. 